\title{
Sprachverstehen mit Hörgeräten für zehn Standardaudiogramme
}

Hals-Nasen-Ohren-Klinik, Kopf- und Halschirurgie

\author{
Abteilung Audiologie
}

Der Medizinischen Fakultät der

Friedrich-Alexander-Universität Erlangen-Nürnberg zur

Erlangung des Doktorgrades Dr. med. dent. vorgelegt von: Constantin Claus Dörfler

Erlangen, 2020 


\section{Als Dissertation genehmigt von der Medizinischen Fakultät der Friedrich-Alexander-Universität Erlangen-Nürnberg}

Vorsitzender des Promotionsorgans:

Gutachter:

Gutachter:

Tag der mündlichen Prüfung:
Prof. Dr. med. Markus Neurath

Prof. Dr. Dr. Ulrich Hoppe

Prof. Dr. Michael Döllinger

3. November 2020 
Inhaltsverzeichnis

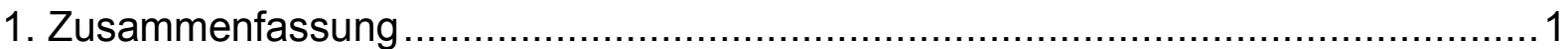

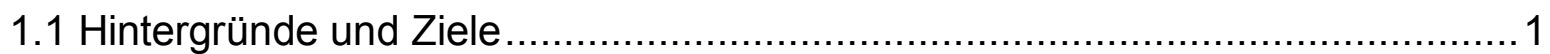

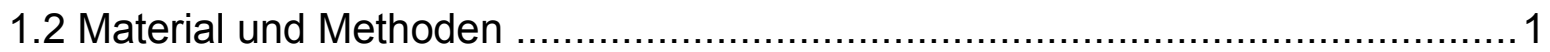

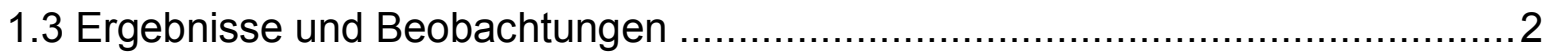

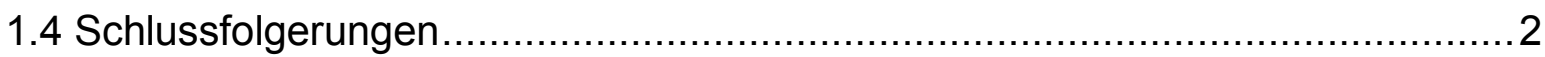

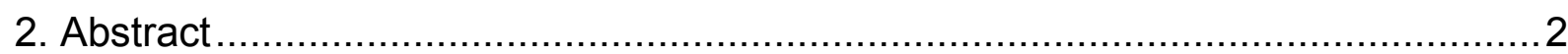

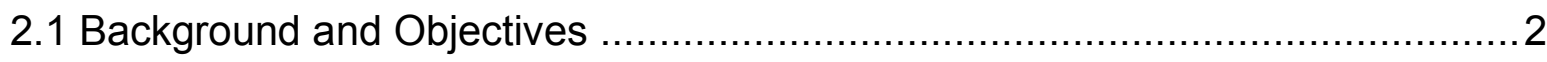

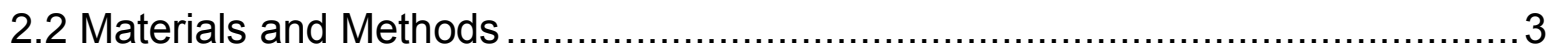

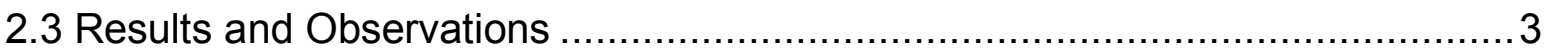

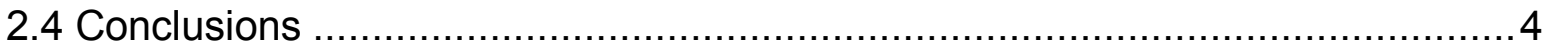

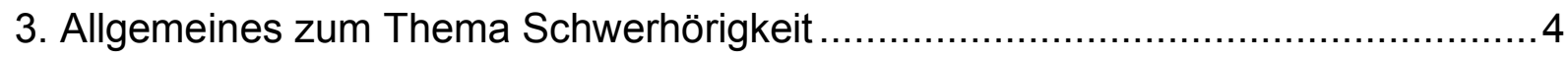

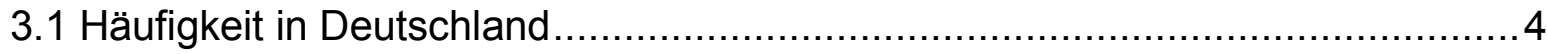

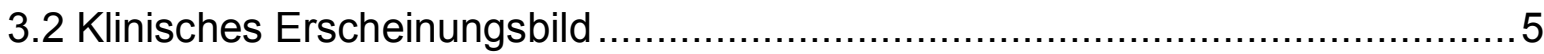

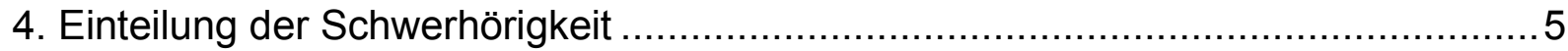

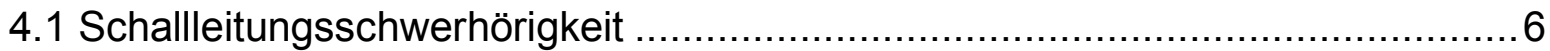

4.2 Schallempfindungs- bzw. sensorische Schwerhörigkeit ..........................6

5. Häufige Ursachen der Schwerhörigkeit nach Lokalisation .............................. 7

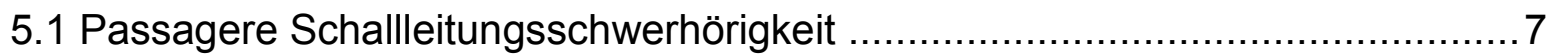

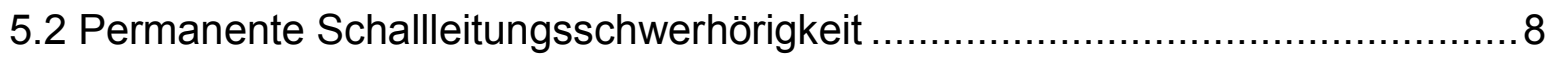

5.3 Schallempfindungsschwerhörigkeit (sensorische Schwerhörigkeit) ............... 8

5.4 Akute Schallempfindungsschwerhörigkeit ...............................................

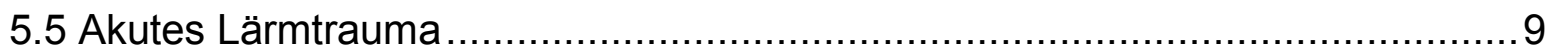

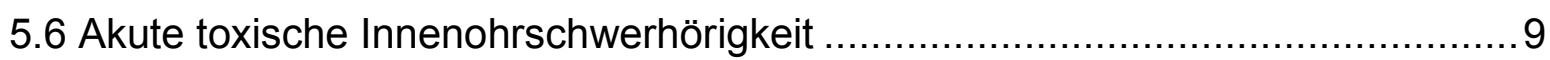

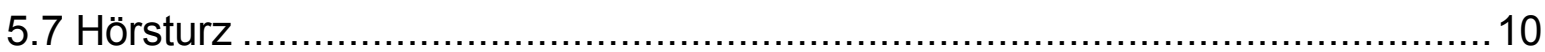


5.8 Permanente Schallempfindungsschwerhörigkeit (Presbyakusis bzw. Alters-

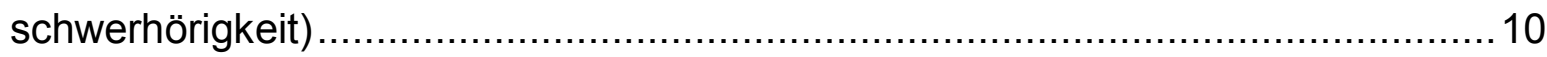

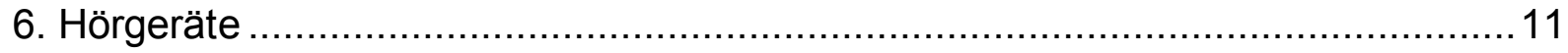

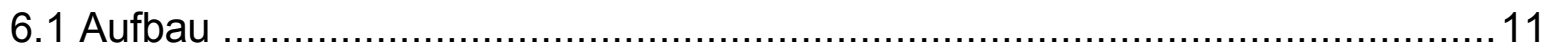

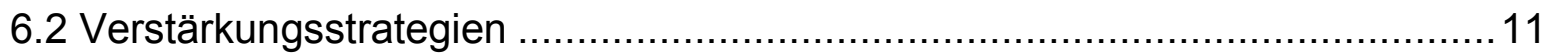

6.3 Verifikation und Validierung von Hörsystemversorgungen ............................ 12

6.4 Standardaudiogramme nach Bisgaard ................................................. 13

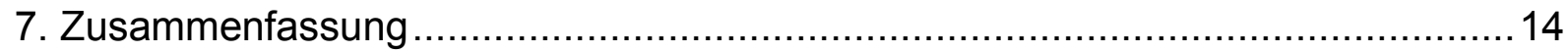

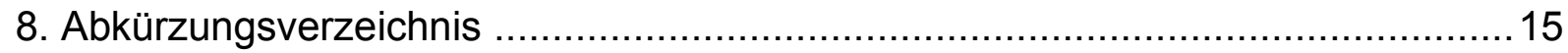

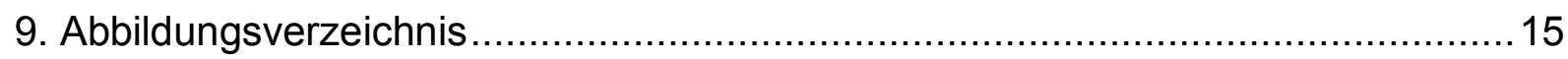

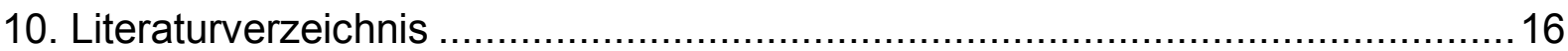

11. Originalpublikation: Sprachverstehen mit Hörgeräten für 10 Standardaudiogramme 11.1 Hintergrund.

11.2 Patienten und Methode.

11.2.1 Patienten

11.2.2 Messungen

11.2.3 Datenanalyse.

11.3 Ergebnisse

11.4 Diskussion

11.4.1 Spezifische Audiogrammverläufe und Sprachverstehen.

11.4.2 Vergleich mit früheren Untersuchungen

11.4.3 Praktische Aspekte der Hörgeräteevaluierung mittels Sprachaudiometrie

11.5 Fazit für die Praxis.

11.6. Einhaltung ethischer Richtlinien

11.7 Literatur. 
12. Danksagung.

13. Lebenslauf.. 


\section{Zusammenfassung}

\subsection{Hintergründe und Ziele}

Die Hörfähigkeit ist maßgeblich für die sprachliche, emotionale und geistige Entwicklung des Menschen verantwortlich und ist im alltäglichen Leben von immenser Bedeutung.

In Deutschland leiden ca. 10-12 Millionen Menschen an einer behandlungsbedürftigen innenohrbedingten Schwerhörigkeit [21]. Schwerhörigkeit kann zu einer maßgeblichen Beeinträchtigung der Lebensqualität der Betroffenen führen [13]. Im Falle einer Schwerhörigkeit ist in der Regel eine Hörgeräteversorgung indiziert. Hierbei wird in erster Linie eine Verbesserung des Sprachverstehens in Ruhe und vor allem im Störgeräusch angestrebt [5,11]. Von großer Bedeutung ist neben der Indikationsstellung auch die Evaluierung der Hörgeräteversorgung durch den HNOArzt $[10,12,18]$. Der Versorgungserfolg variiert in der Praxis jedoch stark.

Ziel dieser Arbeit war die Untersuchung des Zusammenhangs zwischen Ausmaß und Art des Hörverlustes (Audiogrammtyp), maximalem Einsilberverstehen und Sprachverstehen mit Hörgerät.

\subsection{Material und Methoden}

Im Zuge dieser retrospektiven Studie wurden die ton - und sprachaudiometrischen Werte von 740 Ohren von 370 Patienten, die sich zwischen 2012 und 2017 in der HNO-Klinik der Friedrich-Alexander-Universität Erlangen-Nürnberg (FAU) zur Kontrolle ihrer Hörgeräteversorgung vorgestellt hatten, ausgewertet. Insgesamt wurden 2357 Hörgeräteüberprüfungen untersucht. In die Auswahl wurden beidseitig versorgte Hörgeräteträger mit mindestens drei Monaten Hörgeräteerfahrung, Deutsch als Muttersprache und vollendetem 18. Lebensjahr integriert. Analysiert wurden dabei das maximale Verstehen im Freiburger Einsilbertest (mEV) und das Einsilberverstehen mit Hörgerät (EV65(HG)) für zehn unterschiedliche Standardaudiogrammtypen.

Der Frequenzbereich für die Messung der Luftleitungsschwellen lag zwischen 0,125 und $8 \mathrm{kHz}$, die der Knochenleitungsschwellen zwischen 0,25 und 6kHz. Mithilfe des Freiburger Einsilbertest wurde das Sprachverstehen der Probanden ohne Hörgeräteversorgung analysiert. Zu Beginn wurde hierbei die Messung in Ruhe bei 65 $\mathrm{dB} S \mathrm{SL}$ in monauraler Wiedergabe per Kopfhörer durchgeführt. 
Im Anschluss daran wurde die Lautstärke der Darbietung so erhöht, dass vollständiges Sprachverstehen der Patienten oder ein gerade noch tolerierbarer Pegel (120 dB SPL Audiometergrenze) resultierten.

\subsection{Ergebnisse und Beobachtungen}

Die Differenz zwischen EV65(HG) und mEV liegt zwischen 10-20 Prozentpunkten. Diese Fehlergrenzen stimmen mit denen aus vorherigen Untersuchungen überein. Bei flachen und mäßig abfallenden Hörverlusten ist die Differenz tendenziell größer als bei steil abfallenden Audiogrammen. Der Quotient EV65(HG)/mEV kann als Wirkungsgrad der Hörgeräteversorgung interpretiert werden, da er das tatsächlich erreichte Sprachverstehen mit Hörgerät auf das maximal erreichbare Einsilberverstehen des Schwerhörigen bezieht.

\subsection{Schlussfolgerungen}

Durch eine Hörgeräteversorgung kann nicht in allen Hörverlustklassen eine vergleichbar deutliche Verbesserung der Hörleistung erzielt werden. Von entscheidender Bedeutung sind hierfür das maximale Einsilberverstehen, das Ausmaß und der Frequenzverlauf des Hörverlustes. Auf diesen Umstand muss der Betroffene hingewiesen werden, um eine realistische Erwartungshaltung bezüglich des Versorgungserfolges zu erreichen.

\section{Abstract}

\subsection{Background and Objectives}

Hearing is largely responsible for the linguistic, emotional and spiritual development of people and is of immense importance in everyday life.

In Germany, around 10-12 million people suffer from hearing loss due to treatment of the inner ear [21]. Hearing loss can have a significant impact on the quality of life of those affected [13]. In the case of hearing loss, hearing aid care is usually indicated. This is primarily an improvement in understanding speech at rest and $v$. a. aimed for in noise [5,11]. In addition to the indication, the evaluation of the hearing aid supply by the otolaryngologist is of great importance $[10,12,18]$. However, the success of care varies widely in practice. 
The aim of this work was to investigate the relationship between the extent and type of hearing loss (audiogram type), maximum monosyllabic comprehension and speech understanding with a hearing aid.

\subsection{Materials and Methods}

In the course of this retrospective study, the sound and speech audiometric values of 740 ears from 370 patients who had presented to the ENT clinic of the FriedrichAlexander University Erlangen-Nürnberg (FAU) between 2012 and 2017 to check their hearing aid supply were evaluated. A total of 2357 hearing aid reviews were examined. Both-sided hearing aid users with at least three months of hearing aid experience, German as their mother tongue and over the age of 18 were integrated into the selection. The maximum understanding in the Freiburg monosyllabic test (mEV) and the monosyllabic understanding with hearing aid (EV65 (HG)) for ten different standard audiogram types were analyzed.

The frequency range for the measurement of the air conduction thresholds was between 0.125 and $8 \mathrm{kHz}$, that of the bone conduction thresholds between 0.25 and 6 $\mathrm{kHz}$. With the help of the Freiburg Monosyllabic Test the speech understanding of the test persons was analyzed. At the beginning, the measurement was carried out at rest at $65 \mathrm{~dB}$ SPL in monaural reproduction using headphones. Subsequently, the volume of the performance was increased so that complete speech understanding of the patient or a level that was just tolerable (120 dB SPL audiometer limit) resulted.

\subsection{Results and Observations}

The difference between EV65 (HG) and mEV is between 10-20 percentage points. These error limits agree with those from previous studies. With flat and moderately decreasing hearing losses, the difference tends to be larger than with steeply decreasing audiograms. The quotient EV65 (HG) / mEV can be interpreted as the efficiency of the hearing aid supply because it relates the speech understanding actually achieved with the hearing aid to the maximum achievable monosyllabic understanding of the hearing impaired. 


\subsection{Conclusions}

A hearing aid supply cannot achieve a comparable significant improvement in hearing performance in all hearing loss classes. The maximum understanding of monosyllables, the extent and frequency of the hearing loss are of crucial importance. The affected person must be informed of this in order to achieve realistic expectations regarding the success of care.

\section{Allgemeines zum Thema Schwerhörigkeit}

Unter Schwerhörigkeit (Hypakusis) wird eine Verminderung der Hörfähigkeit in unterschiedlichem Ausmaß verstanden. Sie kann von einer leichten, nicht therapiebedürftigen Schwerhörigkeit bis zur Gehörlosigkeit reichen. Ätiologisch kommen alle Ebenen des Hörorgans in Frage. Diese reichen von der Schallleitung zum Innenohr, über die Schallempfindung durch die Sinneszellen der Cochlea, bis hin zur Schallverarbeitung entlang des Hörnervs, der Hörbahn oder der Hörzentren [23].

Somit ist Schwerhörigkeit ein Symptom welches die Erkrankung des Hörorgans als Ursache hat. An dieser Stelle muss gegenüber anderen Formen der Hörstörung wie zum Beispiel der Hyperakusis einer Überempfindlichkeit gegenüber Schall, dem fluktuierenden Gehör oder dem Tinnitus differenziert werden [23].

\subsection{Häufigkeit in Deutschland}

Die Schwerhörigkeit ist in den Industrieländern eine der fünf häufigsten Erkrankungen, die mit einer wesentlichen Beeinträchtigung der Lebensqualität der Betroffenen einhergeht [13]. Laut der Weltgesundheitsorganisation (WHO) beginnt Schwerhörigkeit ab einem gemittelten Hörverlust von $25 \mathrm{~dB}$ [23].

Um diesen zu ermitteln, werden dem Probanden Töne in steigender Lautstärke vorgespielt, bis der Proband diese wahrnimmt. Durch mehrmaliges Wiederholen wird so die genaue Hörschwelle bestimmt. Die zugrundeliegenden Frequenzen sind 500 $\mathrm{Hz}$ sowie 1,2 und $4 \mathrm{kHz}$ [22]. Bis zu einem Hörverlust von $40 \mathrm{~dB}$, liegt eine geringgradige Schwerhörigkeit vor, diese äußert sich für den Betroffenen darin, dass Umgangssprache in einem Umkreis von $1 \mathrm{~m}$ vom Ohr entfernt verstanden wird. Bei Hörverlusten von 40-60 dB, handelt es sich um eine mittelgradige Schwerhörigkeit. Im klinischen Kontext versteht der Proband lautes Sprechen im Umkreis von $1 \mathrm{~m}$. Hochgradige Schwerhörigkeit ist in einem Bereich von 60-80 dB definiert. 
Hier werden nur noch einzelne Wörter bei sehr lautem Sprechen verstanden. Hörverluste von mehr als $80 \mathrm{~dB}$ werden als Ertaubung klassifiziert, wobei klinisch keinerlei Sprachverständnis trotz maximaler Lautstärke zu erzielen ist [23]. Bezogen auf Deutschland leiden rund 16\% der Erwachsenen zumeist an einer geringgradigen Schwerhörigkeit. Gemäß epidemiologischer Studien in Deutschland kann man von etwa 10-12 Millionen Erwachsenen ausgehen, die an einer behandlungsbedürftigen Schwerhörigkeit leiden [21], ohne dass derzeit eine kausale Therapie zur Verfügung steht. In diesen Fällen sind in der Regel Hörgeräteversorgungen indiziert. In erster Linie wird durch eine Hörgeräteversorgung ein möglichst weitgehender Ausgleich des Sensitivitätsverlustes angestrebt [5,11]. Betrachtet man zudem die demographische Entwicklung ist davon auszugehen, dass sich die Zahl der Schwerhörigen laut WHOKriterien um jährlich etwa 150.000-160.000 Erwachsene in Deutschland erhöht. Dies geht mit einem Prävalenzanstieg von 1\% pro Jahrfünft einher [3].

\subsection{Klinisches Erscheinungsbild}

Eine beginnende Schwerhörigkeit wird vom Betroffenen meist durch profane Verhaltensmuster kompensiert. Zu Beginn wird der Hörverlust dadurch kaschiert, dass Audioquellen wie zum Beispiel Radio oder Fernseher lauter gestellt werden oder bei einseitiger Schwerhörigkeit das besser hörende Ohr der Schallquelle zugewandt wird. Bei länger andauernder und zunehmender Schwerhörigkeit gewinnt der visuelle Sinn für die korrekte Spracherkennung an Bedeutung, was im allgemeinen Sprachgebrauch als Lippenlesen bezeichnet wird. Andere Auffälligkeiten sind das wiederholte Nachfragen, inhaltlich falsche Beantwortung von Fragen oder eine zu laute Sprechstimme [23].

\section{Einteilung der Schwerhörigkeit}

Schwerhörigkeit ist in ihrer Systematik nicht einheitlich. Um den Begriff der Schwerhörigkeit greifbarer zu machen, muss grundsätzlich nach verschiedenen Parametern differenziert werden. Unter anderem lassen sich Einteilungen nach der Lokalisation im Hörsystem, der Art der Signalverarbeitung, dem Schweregrad, dem zeitlichen Verlauf, dem Verlauf der Hörschwelle im Reintonaudiogramm, dem Patientenalter und der auslösenden Ursache unterscheiden. Teilweise gehen die verschiedenen Parameter ineinander über [10,24]. 
Die Einteilung nach dem Schweregrad anhand des Reintonaudiogrammes und die grundlegende topografisch-funktionelle Differenzierung zwischen Schallleitungsstörung, Schallempfindungsstörung und Schallverarbeitungsstörung sind vor allem im klinischen Gebrauch verbreitet. In Addition dazu existieren die Einteilungen nach dem Patientenalter, beispielsweise die Differenzierung nach kindlicher Schwerhörigkeit und Altersschwerhörigkeit sowie die Einteilung nach dem zeitlichen Verlauf der Schwerhörigkeit und dem Frequenzverlauf der Hörschwelle [10,24]

\subsection{Schallleitungsschwerhörigkeit}

Bei einer Schallleitungsschwerhörigkeit handelt es sich um eine Störung der akustomechanischen Schallausbreitung. Grundsätzlich gelangen Schallwellen über die Ohrmuschel und den Gehörgang an das menschliche Trommelfell. Dieses wird wie eine Mikrofonmembran in mechanische Schwingungen versetzt. Anschließend werden diese Schwingungen über die Gehörknöchelchenkette an die Peri- und Endolymphe der Cochlea übertragen. Störungen auf dem Weg der Schalleitung lassen sich als Schalleitungsschwerhörigkeit zusammenfassen und sind mechanischer Natur [23].

\subsection{Schallempfindungs- bzw. sensorische Schwerhörigkeit}

Bei einer Schallempfindungs- oder auch sensorischen Schwerhörigkeit handelt es sich um eine Störung der elektromechanischen Transduktion durch die inneren und äußeren Haarzellen (Neuroepithel der Cochlea) sowie der Umwandlung in ein Hörnervensignal [25].

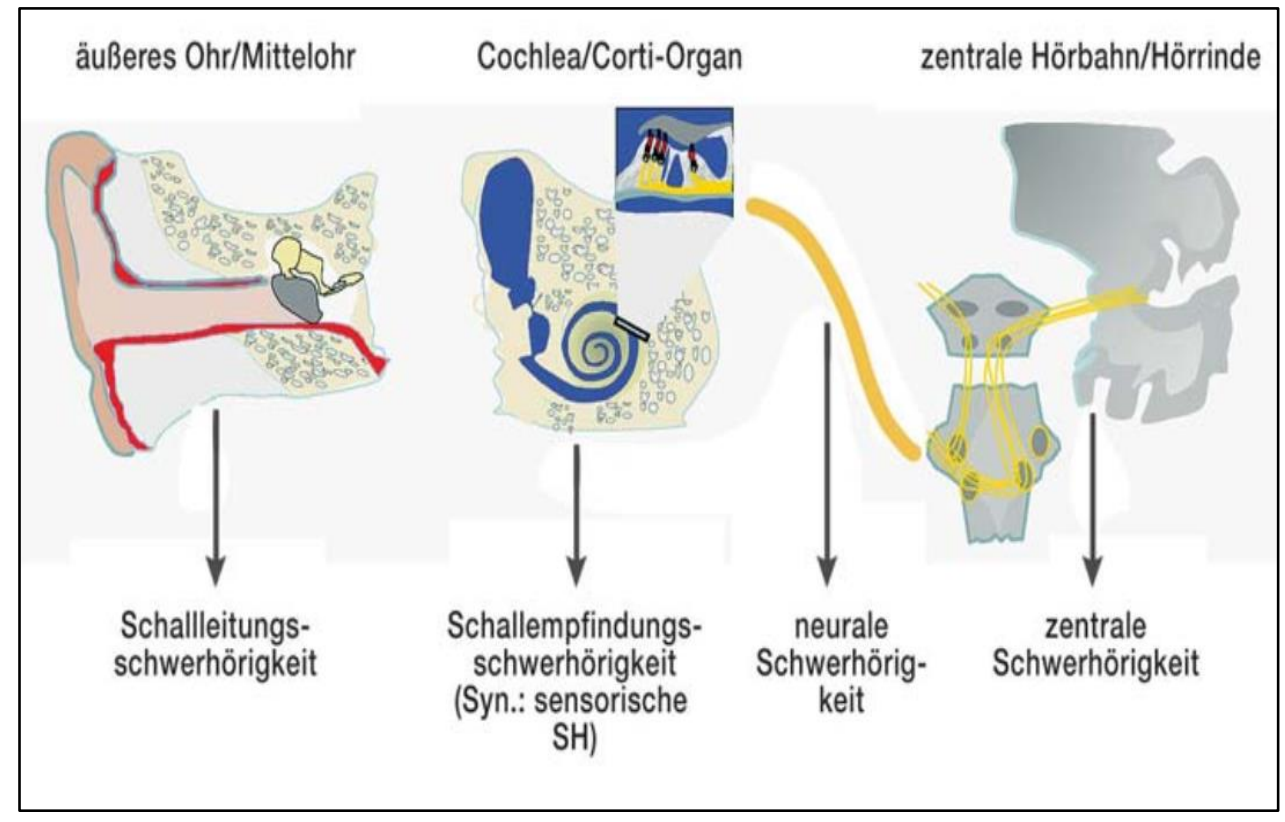


Abbildung 1: Verschiedene Formen der Schwerhörigkeit und deren topographischer Bezug [23:435]

Durch Körperschallausbreitung ergibt sich ein zweiter Weg. So kann die Basilarmembran entweder durch die Steigbügelfußplatte in Schwingung versetzt werden oder äquivalent durch akustisches Anregen des Schädelknochens, beispielweise durch das Aufsetzen einer Stimmgabel [23]. „Diesen Weg der Schallausbreitung zum Innenohr bezeichnet man als Knochenleitung. Bei Störung der Knochenleitungswahrnehmung liegt die Störung im Bereich der Cochlea selbst oder den nachgeschalteten Abschnitten der Hörbahn“ [23:436].

Ist der Nervus cochlearis betroffen, spricht man von neuraler Schwerhörigkeit. Bei einer Beschädigung der zentralen Hörbahn bzw. der Hörrinde handelt es sich um eine zentrale Schwerhörigkeit. Ist die Cochlea und der Hörnerv beteiligt, wird oft von sensorineuraler Schwerhörigkeit gesprochen [23].

Im Folgenden sollen nun die Ursachen einer Hörminderung verdeutlicht werden.

5. Häufige Ursachen der Schwerhörigkeit nach Lokalisation

\subsection{Passagere Schallleitungsschwerhörigkeit}

Eine Schallleitungsschwerhörigkeit entsteht durch Verlegung des äußeren Gehörgangs. In der Klinik imponiert eine solche Schwerhörigkeit beispielsweise durch einen obturierende Zeruminalpfropf oder eine Gehörgangsatresie. Hierbei kann die Hörschwelle um bis zu $60 \mathrm{~dB}$ verschoben sein [23].

Liegt die Störung im Bereich des Trommelfells, der Paukenhöhle und der Gehörknöchelchenkette, ist eine passagere Schallleitungsschwerhörigkeit die Folge [23].

Verschließt sich die Tuba eustachii, entsteht innerhalb von zwei Stunden ein Unterdruck in der Paukenhöhle von $165 \mathrm{mmWs}$ (daPa) [14]. Dieser mindert die Schwingung des Trommelfells vor allem in den tiefen Frequenzen [2].

Eine über Monate andauernde Tubenblockade, erzeugt einen mukoserösen Paukenerguss. Übertragungsverluste von bis zu $40 \mathrm{~dB}$ erstrecken sich hierbei über den gesamten Frequenzbereich. Dieses Krankheitsbild tritt häufig bei Kindern auf. 
Grund dafür sind die engen anatomischen Verhältnisse und die höhere Anfälligkeit für Infekte. Dies hat zur Folge, dass 10 - 30\% der Kinder zwischen dem ersten und dritten Lebensjahr einen Paukenerguss entwickeln und daraus eine Schallleitungsstörung resultiert [4].

Deutlich häufiger sind Kinder mit einer Lippen-, Kiefer-, Gaumenspalte, Trisomie 21 oder dem Ullrich-Turner-Syndrom betroffen. Hierbei entstehen Fehlbildungen des Gaumens und der Tube. Persistiert ein Paukenerguss über drei Monate, sollte auch um Sprachentwicklungsstörungen zu vermeiden, eine Paukendrainage und gegebenenfalls eine Adenotomie in Betracht gezogen werden [20].

\subsection{Permanente Schallleitungsschwerhörigkeit}

Im Gegensatz zur passageren Schallleitungsschwerhörigkeit steht die permanente Schallleitungsschwerhörigkeit. Sie entwickelt sich durch eine chronische Entzündung des Mittelohres. Im kausalen Zusammenhang stehen hierbei eine chronische Schleimhauteiterung (Otitis media mesotympanalis) oder eine chronische Knocheneiterung (Cholesteatom). Ursächlich für die Schwerhörigkeit ist hierbei die Dämpfung (Granulation, Cholesteatom), Zerstörung (enzymatischer Abbau) oder entzündliche Fixation von Trommelfell und Ossikelkette. Das Maß der Schwerhörigkeit (30-60 dB) gibt in den meisten Fällen keinen Rückschluss auf den Grad der Destruktion an, da das inflammatorische Gewebe innerhalb der Paukenhöhle ebenfalls Schwingungen überträgt und somit Mängel akustisch kaschieren kann [17].

In diesem Fall ist die Operation das Mittel der Wahl mit dem Ziel der vollständigen Eliminierung der Krankheitserreger, sowie der Wiederherstellung der Gehörknöchelchenkette [23].

\subsection{Schallempfindungsschwerhörigkeit (sensorische Schwerhörigkeit)}

Die Umwandlung der mechanischen Perilymphschwingung in ein elektrisches Nervenpotenzial geschieht im Cortischen Organ [23].

Die Schwingung der Basilarmembran hat zur Folge, dass die Stereozilien mechanisch ausgelenkt werden. Das führt zu einem Elektrolyteinstrom in den Haarzellen und einer damit verbundenen Depolarisation. Motorproteine in den Zellwänden der äußeren Haarzellen sorgen dafür, dass der elektromechanische Transduktionsprozess in einem gewissen Dynamikbereich nichtlinear verstärkt werden [25]. 
Diese Funktion ist bei einer Schallempfindungsschwerhörigkeit beeinträcht. Zum größten Teil sind hier die äußeren Haarzellen betroffen. Daraus resultiert der Abfall der Knochenleitungsschwelle bis $50 \mathrm{~dB}$, das Defizit der nichtlinearen Verstärkung (Recruitmentphänomen) und der begrenzten Frequenzselektivität (Verzerrungen) [23].

\subsection{Akute Schallempfindungsschwerhörigkeit}

Eine Schädigung der Sinneszellen oder der Homöostase des Innenohrs ziehen akute Hörstörungen nach sich. Die Gründe hierfür können vielfältig sein. Neben einem Trauma (z.B. Schädelbasisfraktur oder Lärmtrauma), kann die Ursache auch toxischinfektiös oder idiopatisch sein [23].

Das Knalltrauma ist charakterisiert durch eine kurzzeitige Schallbelastung über $140 \mathrm{~dB}$ und die sehr kurze Dauer des Druckanstiegs ( $<1,5 \mathrm{~ms}$ ). Bei einem Explosionstrauma wiederum dauert der Druckanstieg deutlich länger (>2 ms) und eine Trommelfellruptur ist die Folge [23]. Signifikant ist in beiden Fällen eine sofort eintretende, meist über Stunden anhaltende Hörminderung mit Tinnitus [15].

\subsection{Akutes Lärmtrauma}

Wirkt hingegen Lärm mit relativ hohen Pegeln über einen längeren Zeitraum beispielsweise bei einem Rockkonzert ein, spricht man von einem akuten Lärmtrauma. Im Vordergrund stehen dabei schwere metabolische Störungen (oxidativer Stress), die je nach Dauer auch irreversibel sein können. Symptomatisch ist außerdem ein Hochtonverlust um $4 \mathrm{kHz}$ und ein Tinnitus in diesem Frequenzbereich. Behandelt werden solche akuten Schallempfindungsstörungen durch Infusionen mit Rheologika und Kortison [23].

\subsection{Akute toxische Innenohrschwerhörigkeit}

Über die Rundfenstermembran, den Liquor oder die Blutversorgung können sowohl Medikamente als auch bakterielle und virale Toxine an das Innenohr gelangen und die Haarzellen irreversibel schädigen. Medikamente, die am häufigsten mit der toxischen Innenohrschwerhörigkeit in Verbindung gebracht werden, sind Aminoglykoside, Zytostatika, Schleifendiuretika, Salizylate und Chinin. In der heutigen Medizin wird durch Kontrolle der Serumkonzentrationsspiegeln das Risiko der Haarzellschädigung durch Medikamente reduziert. 
Bakterientoxine beziehungsweise Entzündungsmediatoren bei Virusinfekten wirken ebenfalls toxisch auf das Innenohr (Labyrinthitis). Zu nennen ist die toxische Labyrinthitis im Rahmen einer Grippeotitis, einer eitrigen Meningitis oder der chronischen Otitis media [16].

\subsection{Hörsturz}

Unter dem Begriff Hörsturz wird eine ex abrupto auftretende Hörstörung charakterisiert, die nicht ausschließlich durch das Innenohr hervorgerufen wird. Im englischen Sprachgebrauch wird der Begriff "Sudden Sensorineural Hearing loss“ verwendet [1].

Der Hörsturz kann bei einer Vielzahl von Krankheiten symptomatisch auftreten:

- Infektionen in 12,8\% (Meningitits, Lues oder HIV-Infektion)

- Ohrerkrankungen in 4,7\% (Cholesteatom)

- Trauma in 4,2 \% (Knalltrauma, Schädelbasisfraktur)

- Kardiovaskulär in 2,8\%

- Paraneoplastisch in 2,2 \%

Im Großteil der Fälle findet sich trotz intensiver Stufendiagnostik keine erkennbare Ursache (idiopathischer Hörsturz). Vermutet wird, dass eine unbekannte virale, vaskuläre oder immunologische Genese ursächlich für eine Homöostasestörung des Innenohres ist [19].

\subsection{Permanente Schallempfindungsschwerhörigkeit (Presbyakusis bzw.}

\section{Altersschwerhörigkeit)}

Eine Permanente Schallempfindungsstörung ist definiert durch eine sensorineurale, beidseitige Schwerhörigkeit, die durch viele Faktoren bedingt ist. Sie tritt ab dem 5 . bis 6. Lebensjahrzehnt auf und zeichnet sich reintonaudiometrisch durch einen Hochtonverlust aus. Als Ursache werden physiologische Alterungsprozesse in der mikrovaskulären Versorgung der Haarzellen (sensorischer Typ) diskutiert, welche Ischämie, Hypoxie und oxidativen Stress zur Folge haben. Des Weiteren können die Ganglienzellen (neuronaler Typ) oder die Stria vascularis (metabolischer Typ) dabei beteiligt sein. Schätzungsweise sind etwa $40 \%$ der genannten Bevölkerungsgruppe von Altersschwerhörigkeit betroffen [17]. 
Trotz der hohen Prävalenz sind bis dato keine Präventionsoptionen bekannt. Um die Lebensqualität der Betroffenen zu verbessern, wird ab einem Hörschwellenverlust von $30 \mathrm{~dB}$ in den Sprachfrequenzen eine Hörgeräteversorgung empfohlen. Liegt ein nahezu vollständiger Verlust des Sprachverständnisses vor, sind Cochlea-Implantate indiziert [23].

\section{Hörgeräte}

\subsection{Aufbau}

Heutige digitale Hörgeräte empfangen das Eingangssignal über ein Doppelmikrofonsystem mit vorderer und rückwärtiger Schalleintrittsöffnung. Somit ist durch Verrechnung beider Signale der omnidirektionale, als auch der Betrieb mit verschiedenen Richtcharakteristiken möglich. Damit elektromagnetische Wellen zum Beispiel von Ringschleifen (in Kirchen, Kinos, Theatern oder anderen öffentlichen Gebäuden) oder Telefonhörern induktiv aufgenommen werden können, verfügen Hörgeräte zusätzlich über einen Empfänger (Induktionsspule, Telefonspule). Zudem ist es mit modernen Hörgeräten möglich, eine Verbindung zu externen Audioquellen wie Smartphones, Tablets oder Fernsehgeräten über ein integriertes Wireless-Modul herzustellen. Somit lassen sich nicht nur Audiosignale in hoher Qualität streamen, sondern auch gleichzeitig der Betriebszustand des Geräts fortlaufend synchronisieren und das Gerät kabellos programmieren [8].

Mit Hilfe eines A/D-Wandlers (analog/digital) wird das elektrische Eingangssignal, das vom Mikrofonsystem, der Induktionsspule oder dem Wireless-Modul kommt, für die Verarbeitung im DSP (Digital Signal Processor) digitalisiert und in Frequenzbänder zerlegt. Im Anschluss daran wird das Signal in den einzelnen Frequenzbändern abhängig vom Eingangspegel sowie anderen Signalparametern entsprechend der Bedürfnisse des Nutzers verarbeitet [8]. „Neben Verstärkungs- und Kompressionsfunktion verfügen aktuelle Hörgerätesysteme über zahlreiche weitere Signalverarbeitungsstrategien und Features, wie z.B. Rückkopplungs- und Störschallunterdrückung usw. [...]. [8:75].

\subsection{Verstärkungsstrategien}

Nach Kießling ist die zentrale Aufgabe der Hörgeräte, eine lautheitsgerechte Wahrnehmung der Umgebungsgeräusche für den Nutzer zu ermöglichen. 
Dabei richtet man sich nach dem Bereich der Restdynamik des Nutzers. So sollen weiterhin leise und laute Eingangssignale laut wiedergegeben werden [8].

Kießling beschreibt weiter, dass eine lineare Verstärkung der Signale dann möglich ist, wenn der Restdynamikbereich ausreichend breit ist. Somit werden vom Hörgerät sämtliche Frequenzbänder einheitlich verstärkt. Dies ist jedoch in den seltensten Fällen gegeben. Weitaus häufiger sind frequenzabhängige Hörverluste mit frequenzspezifischer eingeengter Restdynamik. In diesen Fällen ist das Prinzip der nichtlinearen Verstärkung indiziert. Hierbei wird das Eingangssignal in verschiedene Frequenzbänder zerlegt und die Verstärkung bzw. Kompression individuell an den jeweiligen Hörverlust des Patienten angepasst. Durchschnittliche Hörgeräte verfügen über wenige Frequenzbänder, in denen das Verstärkungs- und Kompressionsverhalten eingestellt werden kann. Hochwertige Hörgeräte haben hingegen 20 und mehr Frequenzbänder um das Verstärkungs- und Kompressionsverhalten individuell und differenziert auf die Bedürfnisse des Nutzers anzupassen [8].

\subsection{Verifikation und Validierung von Hörsystemversorgungen}

„Die Hörgeräteverifikation soll überprüfen, ob die Zielvorgaben bezüglich Frequenzgang und Ausgangsschalldruckpegel verwirklicht wurden [...]" [9:148]. Hierbei wird ebenso die korrekte Verarbeitung des Hörgerätesignals geprüft, die u.a Verstärkung, Kompression und Unterdrückung des Störschalls beinhaltet. Die Messungen und Untersuchungen im Rahmen der Verifikation finden in der Regel in der Anpassungsumgebung bei Hörgeräteakustiker mit und ohne aktive Mitteilung des Hörgerätenutzers statt [9].

„Typische Verifikationsverfahren sind, Sondenmikrofonmessung, Hörschwellenbestimmung und Lautheitsskallierung mit angepassten Hörgeräten“ [9:148]. Bei beiden Verfahren wird die jeweilige Hörgerätewiedergabe, entweder am Kuppler oder direkt am Ohr, mit der zu erwartenden Zieleinstellung abgeglichen. Es lässt sich jedoch weder auf eine gute Akzeptanz noch auf ein ideales Sprachverstehen schließen. Deswegen sollte das Ziel einer Hörgeräteanpassung nicht sein, möglichst nahe an der Zielwiedergabe zu liegen, sondern vielmehr eine Verbesserung für den Nutzer im Alltag hervorzurufen [9]. 
„Aufsetzend auf die Verifikation erfolgt die Validierung des Hörgerätenutzens im Anpassraum sowie im Alltag“ [9:149]. Um mithilfe der Hörgerätevalidierung den Gewinn und Mehrwert der Hörgeräte zu beschreiben, eignen sich neben Fragebögen oder Hörtagebücher auch Sprachtests in einer Hörkabine. Bei Letzterem ist darauf zu achten, dass die Ergebnisse lediglich hinweisenden, aber nicht beweisenden Charakter besitzen. Falls sich bei der Validierung Defizite der Versorgung offenbaren, ist eine weitere Feinanpassung erforderlich [9].

\subsection{Standardaudiogramme nach Bisgaard}

Bisgaard et al. haben eine Kategorisierung von Tonaudiogrammen für die Hörgeräteindustrie in zehn Klassen vorgeschlagen. Diese basiert auf einer statistischen Analyse von über 20.000 Audiogrammen. Im Gegensatz zur WHOEinteilung nach Mittelwerten bei $0,5,1,2$ und $4 \mathrm{kHz}$ werden dabei auch der Frequenzverlauf berücksichtigt.

Die Einteilung der Audiogramme war motiviert durch die Anforderungen der DIN EN 60118-15. Eine realitäsnahe Charakterisierung der Hörgeräte und deren Einstellung ist hierbei von besonderer Bedeutung. Es ist naheliegend, diese Standardaudiogramme auch auf klinische Fragestellungen, die mit der Versorgung von Hörgeräten verbunden sind, einzusetzen.

Alle Standardaudiogramme aus Abb. 1. weisen einen mit steigender Frequenz zunehmenden Hörverlust auf. Gegliedert sind diese in Audiogramme mit einer geringen bis mittleren Frequenzabhängigkeit ( $\mathrm{N}-\mathrm{Typ}$ ) und andere mit starker Frequenzabhängigkeit (S-Typ). Der $\mathrm{N}$-Typ beschreibt einen maximalen Frequenzabfall von $20 \mathrm{~dB}$ pro Oktave, während der S-Typ einen Abfall von mindestens $25 \mathrm{~dB}$ pro Oktave enthält. Weiter wurde der S-Typ in drei Untertypen und der N-Typ in sieben Untertypen untergliedert. Diese richten sich nach dem Grad des Hörverlustes.

Abb. zeigt unter (a) die flach bis mäßig fallenden und unter (b) die steil abfallenden Audiogramme nach Bisgaard. Links neben den Reintonaudiogrammen werden die einzelnen Typen (N1 bis N7 bzw. S1 bis S3) aufgezeigt, während rechts der einzelnen Kurven die erhobenen Fallzahlen abgebildet sind. 


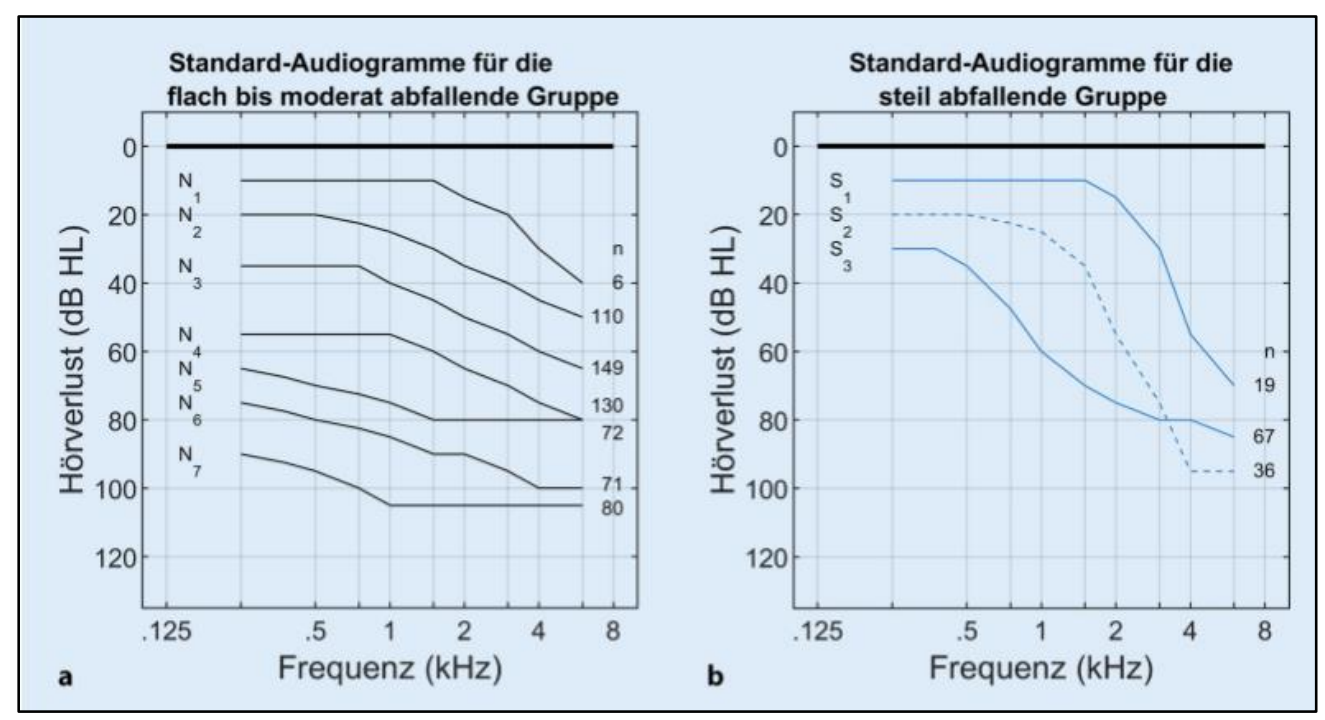

Abbildung 2: Klassifikation der Audiogramme nach Bisgaard

Bisher gibt es noch keine Arbeit, die das Sprachverstehen für diese Audiogrammtypen beschrieben hat.

\section{Zusammenfassung}

Die Fähigkeit zu Hören bedeutet die Sinneswahrnehmung von Schall. Die Hörfähigkeit ist für den Menschen sowohl in der geistigen, sprachlichen und sozialen Entwicklung als auch im alltäglichen Leben von großer Bedeutung. Eine nicht therapierte Beeinträchtigung des Hörvermögens kann für den Betroffenen eine Minderung der Lebensqualität darstellen.

In Deutschland sind laut Gablenz ca. 16\% der Bevölkerung von Schwerhörigkeit, definiert nach den WHO-Kriterien, betroffen.

Durch eine Hörgerätversorgung kann der Funktionsverlust des Hörorgans ausgeglichen und zur Verbesserung des subjektiven Wohlbefindens des Betroffenen beigetragen werden.

Im Zuge der vorliegenden Arbeit kam es zu einer Hörgeräteüberprüfung von 370 Patienten der HNO-Klinik in Erlangen. Ziel war es, die dabei gewonnenen ton- und sprachaudiometrischen Werte miteinander zu vergleichen. Zentral waren, das Sprachverstehen in Ruhe, die Verbesserung der Hörfähigkeit durch eine Hörgeräteversorgung und der Bezug zum maximalen Einsilberverstehen (mEV). 
Der Audiogrammverlauf der untersuchten Fälle wurde in die verschiedenen Standardaudiogramme nach Bisgaard et al. eingeteilt. Es konnte insgesamt festgestellt werden, dass bei flachen und mäßig abfallenden Hörverlusten, die Differenz von mEV und EV65(HG) tendenziell größer ist als bei steil abfallenden Audiogrammen.

Das maximale Einsilberverstehen als auch das Ausmaß und der Frequenzverlauf des individuellen Hörverlust beeinflussen den Erfolg einer Hörgeräteversorgung. Somit können keine allgemeingültigen Vorhersagen über die Verbesserung der Hörleistung getroffen werden. Der Betroffene sollte über diese Zusammenhänge aufgeklärt werden, damit er zu einer realistischen Erwartungshaltung bezüglich des Versorgungserfolgs kommen kann.

\section{Abkürzungsverzeichnis}

\begin{tabular}{ll}
\hline $\mathrm{mEV}$ & maximales Einsilbenverstehen \\
$\mathrm{EV65}(\mathrm{HG})$ & Einsilberverstehen mit Hörgerät \\
$\mathrm{WHO}$ & Weltgesundheitsorganisation \\
$\mathrm{dB}$ & Dezibel \\
$\mathrm{Hz}$ & Hertz \\
$\mathrm{kHz}$ & Kilohertz \\
$\mathrm{mmWS}$ & Millimeter Wassersäule \\
$\mathrm{daPa}$ & Dekapascal \\
$\mathrm{ms}$ & Millisekunden \\
DSP & Digital Signal Processor \\
$\mathrm{HIV}-$ Infektion & Humane Immundefizienz-Virus-Infektion \\
SPL & Sound Pressure Level \\
9. Abbildungsverzeichnis &
\end{tabular}

\begin{tabular}{ll}
\hline Abbildung 1: & Verschiedene Formen der Schwerhörigkeit und deren \\
& topographischer Bezug, [23:435]
\end{tabular}

Abbildung 2: Klassifikation der Audiogramme nach Bisgaard, eigene Darstellung 
1. Chau JK, Lin JR, Atashband S, Irvine RA, Westerberg BD: Systematic review of the evidence for the etiology of adult sudden sensorineural hearing loss. Laryngoscope. 2010; 120: 1011-21.

2. Dai C, Gan RZ: Change of middle ear transfer function in otitis media with effusion model of guinea pigs, Hearing Research 2008; 243: 78-86.

3. Destatis (2013) Ergebnisse der 13. Koordinierten Bevölkerungsvorausberechnung mit Basis 31.12.2013.Variante2mitKontinuitätbeistärkerer Zuwanderung G1-L1W2. Ergebnis 12421-0002. www-genesis.destatis.de. Zugegriffen: 27. Nov 2015.

4. Fiellau-Nikolajsen M: Epidemiology of secretory otitis media. A descriptive cohort study. Ann Otol Rhinol Laryngol. 1983; 92: 172-7.

5. Gemeinsamer Bundesausschuss (2018) Hilfsmittel-Richtlinie über die Verordnung von Hilfsmitteln in der vertragsärztlichen Versorgung (Hilfsmittel Richtlinie/HilfsM$\mathrm{RL}$ ) in der Neufassung vom 19. Juli 2018.BAnz AT 02.10.2018B2.Gemeinsamer Bundesausschuss, Berlin

6. Hoppe U, Hesse G (2017) Hörgeräte: Indikationen, Technologie, AnpassungundQualitätskontrolle. Laryngorhinootologie96(S1):43-65

7. Hüttenbrink KB: Die chronische Otitis media. In: Naumann $\mathrm{H} \mathrm{H}$, Helms J, Herberhold C, Kastenbauer E (eds): Oto-Rhino-Laryngologie in Klinik und Praxis.. Stuttgart: Thieme Verlag, 1994; 601-32.

8. Kießling J. Konventionelle Hörsysteme: Hörgeräte. In: Kießling J, Kollmeier B, Baumann U, Hrsg. Versorgung mit Hörgeräten und Hörimplantaten: 173 Abbildungen. 3., vollständig überarbeitete und erweiterte Auflage. Stuttgart, New York: Georg Thieme Verlag; 2018. S. 73-102.

9. Kießling J. Verifikation und Validierung von Hörsystemversorgungen. In: Kießling J, Kollmeier B, Baumann U, Hrsg. Versorgung mit Hörgeräten und Hörimplantaten: 173 Abbildungen. 3., vollständig überarbeitete und erweiterte Auflage. Stuttgart, New York: Georg Thieme Verlag; 2018. S. 148-59.

10. Lehnhardt E, Laszig R: Praxis der Audiometrie Stuttgart: Georg Thieme Verlag 2009; 47-52.

11. LesicaNA(2018)Whydohearingaidsfailtorestore normal auditory perception. Trends Neurosci 41:174-185. 
12. Löhler J, Akcicek B, Wienke A, Hoppe U (2014) Komplikationen bei der Hörgeräteversorgung ohneHNO-Arzt.HNO62:360-366.

13. Mathers C, Smith A, Concha M (2000) Global burden of hearing loss in the year 2000. Global burden of disease. World Health Organization, Geneva, S130.

14. Pau HW, Sievert U, Just T, Sade J: Pressure changes in the human middle ear without opening the eustachian tube. Acta Oto-Laryngologica 2009; 129: 1182-6.

15. Plontke S K-R, Dietz K, Pfeffer C, Zenner H-P: The incidence of acoustic trauma due to New Year's firecrackers. Eur Arch Otorhinolaryngol 2002; 259: 247-52.

16. Probst R: Innenohr und retrocochleäre Störungen. In: Probst R, Grevers G, Iro H (eds): Hals-Nasen-Ohren-Heilkunde, 3. Aufl. Stuttgart: Thieme 2008; 240.

17. Ries PW: Prevalence and characteristics of persons with hearing trouble: United States, 1990-1991. Series 10: Data From the National Health Survey, No 188, Hyattsville: US Department of Health and Human Services, Publication No. (PHS) 94-1516, 1994; 1-75.

18. Schuchardt W, Löhler J (2012) HörgeräteversorgungohneHNO-ArztHNO60:823826

19. Suckfüll M: Perspectives on the pathophysiology and treatment of sudden idiopathic sensorineural hearing loss. Dtsch Arztebl Int 2009; 106(41): 669-76.

20. S2-Leitlinie: Periphere Hörstörungen im Kindesalter, AWMF-Registernummer 049/010, Stand 02/2005. www.awmf.org/uploads/tx szleit linien/049-010l.pdf

21. Von Gablenz P, Holube I (2015) Prävalenz von Schwerhörigkeit im Nordwesten Deutschlands: Ergebnisse einer epidemiologischen Untersuchung zum Hörstatus (HÖRSTAT).HNO63:195-214.

22. Von Gablenz P. Prävalenz von Schwerhörigkeit in Nord-und Süddeutschland. HNO 2017; (8):663-70.

23.T. Differenzialdiagnose der Schwerhörigkeit. Deutsches Ärzteblatt 2011; 108(25):433-44.

24.Zahnert T: Schwerhörigkeit - Ätiologie, Diagnostik und auditive Rehabilitation. Laryngo-Rhino-Otol 2010; 89(11): 669-93.

25.Zenner HP: The role of outer hair cell damage in the loss of hearing. Ear Nose Throat J 1997; 76: 140, 143-4. 
HNO

https://doi.org/10.1007/s00106-019-00780-5

(c) Springer Medizin Verlag GmbH, ein Teil von Springer Nature 2019

Die Messungen von Hörschwelle und Sprachverstehen sind unverzichtbar für die Beurteilung des Erfolgs einer Hörgeräteversorgung. Hierbei sind die Beziehungen der verschiedenen sprachaudiometrischen Ergebnisse von zentraler Bedeutung für den die Hörgeräteversorgung evaluierenden HNO-Arzt. Ziel der vorliegenden Arbeit ist die Darstellung des Sprachverstehens in Ruhe in Abhängigkeit von unterschiedlichen Typen des Tonaudiogramms. Damit wird dem HNO-Arzt eine erste Einordnung des individuellen Versorgungserfolgs ermöglicht.

\section{Hintergrund}

Die Schwerhörigkeit gehört in den Industrieländern zu den 5 häufigsten Erkrankungen mit substanzieller Beeinträchtigung der Lebensqualität [17]. In Deutschland kann man von etwa 10-12 Mio. Erwachsenen ausgehen, die an einer behandlungsbedürftigen innenohrbedingten Schwerhörigkeit leiden [25], ohne dass derzeit eine kausale Therapie zur Verfügung steht. In diesen Fällen sind in der Regel Hörgeräteversorgungen indiziert. Hierbei wird in erster Linie eine Verbesserung des Sprachverstehens in Ruhe und v. a.im Störgeräusch angestrebt $[5,13]$. Neben der Indikationsstellung ist

Die folgende Arbeit wurde von Constantin Dörfler zur Erlangung des Titels Dr. med. dent. an der Friedrich-Alexander-Universität Erlangen-Nürnberg (FAU) durchgeführt.

The present work was performed by Constantin Dörfler in fulfillment of the requirements for obtaining the degree Dr. med. dent. at the Friedrich-Alexander-University Erlangen-Nürnberg (FAU).

\section{Dörfler $\cdot$ T. Hocke ${ }^{2} \cdot$ A. Hast ${ }^{1} \cdot$ U. Hoppe}

${ }^{1}$ Audiologische Abteilung und $\mathrm{CI}$ Centrum CICERO Hals-Nasen-Ohrenklinik, Universitätsklinikum Erlangen, Erlangen, Deutschland

${ }^{2}$ Cochlear Deutschland GmbH \& Co. KG, Hannover, Deutschland

\section{Sprachverstehen mit Hörgeräten für 10 Standardaudiogramme}

hierbei auch die Evaluierung der Hörgeräteversorgung durch den $\mathrm{HNO}$-Arzt von großer Bedeutung [10, 14, 23].

In einer vorangegangenen Studie [7] wurde der Zusammenhang zwischen unversorgt gemessenen audiometrischen Messgrößen und dem Sprachverstehen mit Hörgeräten untersucht. Das Sprachverstehen in Ruhe wurde dabei als Surrogatparameter für den Versorgungserfolg angenommen. Es ist u. a. abhängig von Hörverlust, Hörgeräteanpassung und Lebensalter [21]. Außerdem wurde gezeigt, dass das Einsilberverstehen mit Hörgerät bei $65 \mathrm{~dB}$ SPL, $\mathrm{EV}_{65}(\mathrm{HG})$, bei mittelund hochgradigen Hörverlusten im Mittel um etwa 20 Prozentpunkte unter dem im Sprachaudiogramm mit Kopfhörer ermittelten maximalen Einsilberverstehen, mEV, liegt. Für eine Stichprobe von $n=181$ Versorgungen wurde ein statistischer Zusammenhang zwischen dem Mittelwert der Hörschwellen bei 0,5; 1; 2 und $4 \mathrm{kHz}$ (4FPTA) und $\mathrm{mEV}$ bzw. $\mathrm{EV}_{65}(\mathrm{HG})$ ermittelt, der durch folgende Gleichungen charakterisiert ist:

$$
m E V[\%]=100 \cdot \frac{e^{\left(\beta_{0}+\beta_{1} \cdot 4 F P T A\right)}}{1+e^{\left(\beta_{0}+\beta_{1} \cdot 4 F P T A\right)}}
$$

mit $\beta_{0}=5,99 \pm 0,08$ und $\beta_{1}=-0,0793 \pm$ 0,0012

$$
\begin{aligned}
& E V_{65}(H G)[\%]= \\
& 100 \cdot \frac{e^{\left(\beta_{0}+\beta_{1} \cdot 4 F P T A\right)}}{1+e^{\left(\beta_{0}+\beta_{1} \cdot 4 F P T A\right)}}
\end{aligned}
$$

mit $\beta_{0}=3,96 \pm 0,06$ und $\beta_{1}=-0,0673 \pm$ 0,0011

In anderen Studien mit hörgeräteversorgten Patienten wurden vergleichbare
Werte für die Parameter $\beta_{0}$ und $\beta_{1}$ gefunden $[12,21]$.

Für eine weiterführende Analyse hinsichtlich des frequenzabhängigen Verlaufs des Hörverlusts bietet sich die von Bisgaard et al. [1] vorgeschlagene Einteilung in 10 Standardaudiogramme an. Diese basiert auf einer statistischen Analyse von 28.244 Hörschwellenmessungen und soll typische Audiogramme von Hörgerätenutzern repräsentieren. Die Einteilung war motiviert durch die Anforderungen der DIN EN 60118-15. Diese fordert eine realitätsnahe Charakterisierung der Hörgeräte und der Hörgeräteeinstellung. Es liegt daher nahe, diese Standardaudiogramme auch auf klinische Fragestellungen, die mit der Hörgeräteversorgung verbunden sind, anzuwenden. Alle in - Abb. 1 dargestellten Standardaudiogramme weisen einen mit steigender Frequenz zunehmenden Hörverlust auf. Sie wurden in solche mit geringer bis mittlerer (N-Typ mit maximalem Abfall von $20 \mathrm{~dB}$ pro Oktave) und mit starker Frequenzabhängigkeit (S-Typ mit maximalem Abfall von mindestens $25 \mathrm{~dB}$ pro Oktave) eingeteilt. Beide Typen wurden jeweils noch nach Ausmaß des Hörverlusts in 7 bzw. 3 Untertypen unterteilt $\left(\mathrm{N}_{1}\right.$ bis $\mathrm{N}_{7}$ und $\mathrm{S}_{1}$ bis $\mathrm{S}_{3}$ ).

Ziel der vorliegenden Studie war es, das Sprachverstehen mit und ohne Hörgerät in Abhängigkeit von Ausmaß und Frequenzverlauf des Hörverlusts für eine große Population von Hörgeräteträgern zu untersuchen. Außerdem wurde untersucht, inwieweit die früher gefundenen Zusammenhänge [7] auch für eine aktuelle Population mit modernen Hörsystemen gelten. 

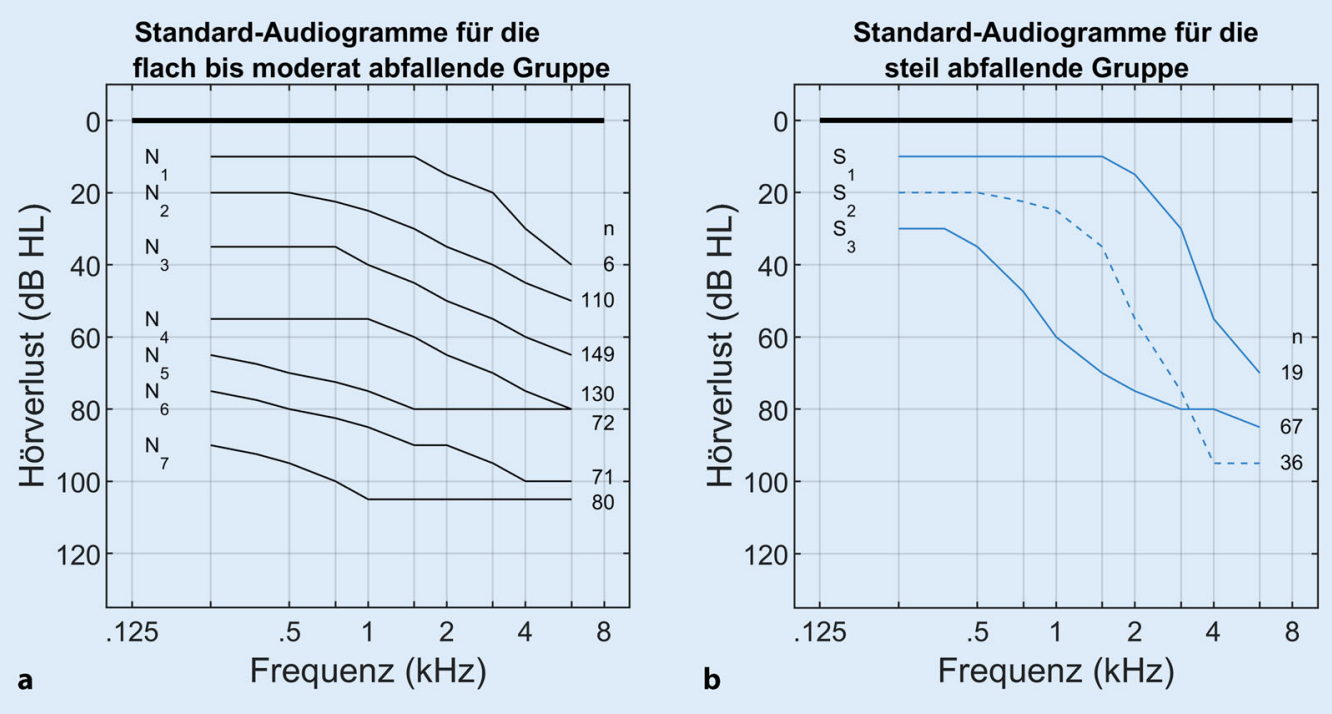

Abb. $1<$ Klassifikation der Audiogramme nach Bisgaard [1]. Bei (a) sind die flach bis moderat fallenden Audiogramme dargestellt. (b) zeigt die steil abfallenden Audiogramme. Die entsprechenden Typen ( $\mathrm{N}_{1}$ bis $\mathrm{N}_{7}$ bzw. $\mathrm{S}_{1}$ bis $\mathrm{S}_{3}$ ) sind links neben den typisierten Reintonaudiogrammen verzeichnet. Rechts der Kurven sind die gefundenen Fallzahlen vermerkt

Tab. 1 Klassifikation der Audiogramme, Gruppengrößen, 4FPTA und Alte

\begin{tabular}{|c|c|c|c|}
\hline \multicolumn{2}{|c|}{ Audiogrammklassifikation u. Gruppengröße } & \multirow{2}{*}{\begin{tabular}{|l|}
$\begin{array}{l}\text { 4FPTA } \\
\text { (dB HL) }\end{array}$ \\
$19 \pm 4$
\end{tabular}} & \multirow{2}{*}{\begin{tabular}{|l}
$\begin{array}{l}\text { Alter (Jahre) } \\
\text { (MW } \pm \text { SD) }\end{array}$ \\
$58 \pm 4$
\end{tabular}} \\
\hline $\mathrm{N}_{1}$ & 6 & & \\
\hline $\mathrm{N}_{2}$ & 110 & $34 \pm 4$ & $65 \pm 11$ \\
\hline $\mathrm{N}_{3}$ & 149 & $47 \pm 4$ & $65 \pm 15$ \\
\hline $\mathrm{N}_{4}$ & 130 & $61 \pm 4$ & $66 \pm 16$ \\
\hline $\mathrm{N}_{5}$ & 72 & $74 \pm 6$ & $62 \pm 18$ \\
\hline $\mathrm{N}_{6}$ & 71 & $89 \pm 5$ & $60 \pm 16$ \\
\hline $\mathrm{N}_{7}$ & 80 & $109 \pm 8$ & $50 \pm 18$ \\
\hline $\mathrm{S}_{1}$ & 19 & $26 \pm 4$ & $62 \pm 23$ \\
\hline $\mathrm{S}_{2}$ & 36 & $46 \pm 7$ & $68 \pm 13$ \\
\hline $\mathrm{S}_{3}$ & 67 & $66 \pm 7$ & $65 \pm 15$ \\
\hline
\end{tabular}

\section{Patienten und Methode}

\section{Patienten}

Im Rahmen dieser retrospektiven Studie wurden 2357 Hörgeräteüberprüfungen ausgewertet, die in der Zeit von 08/2012 bis 09/2017 in der Erlanger HNO-Klinik durchgeführt worden waren. Eingeschlossen wurden beidseitig versorgte Hörgeräteträger mit mindestens $3 \mathrm{Mo}$ naten Hörgeräteerfahrung, Deutsch als Muttersprache und vollendetem 18. Lebensjahr. Die Ausschlusskriterien waren auffällige Otoskopie, eine mittlere Differenz von Luft- und Knochenleitungsschwellen bei 0,$5 ; 1 ; 2$ und $4 \mathrm{kHz}$ von mehr als $5 \mathrm{~dB}$, eine Asymmetrie des 4FPTA auf beiden Seiten von mehr als $20 \mathrm{~dB}$ und technische Defekte an den Hörgeräten.
Es verblieben die Daten von insgesamt 740 Ohren von 370 Patienten (182 Männer, 188 Frauen) im Alter von 21 bis 98 Jahren (Mittelwert: 62,8 Jahre, Standardabweichung: 16,2 Jahre).

\section{Messungen}

Die Luftleitungsschwellen der Reintonaudiometrie wurden für Frequenzen zwischen 0,125 und $8 \mathrm{kHz}$, Knochenleitungsschwellen zwischen 0,25 und $6 \mathrm{kHz}$, gemessen. Zur Messung des Sprachverstehens wurde der Freiburger Einsilbertest verwendet. Die Messungen in Ruhe erfolgten in monauraler Darbietung über Kopfhörer zunächst bei $65 \mathrm{~dB}$ SPL $\left(\mathrm{EV}_{65}\right)$. Anschließend wurde der Präsentationspegel in Schrittweiten von 5 bis $15 \mathrm{~dB}$ erhöht, bis entweder
$100 \%$ Sprachverstehen oder ein gerade noch tolerierbarer Pegel bzw. die Audiometergrenze von $120 \mathrm{~dB}$ SPL erreicht wurde. Die Unbehaglichkeitsschwelle, UCL, entspricht dem kleinsten nicht mehr tolerierten Sprachpegel. Neben dem $\mathrm{EV}_{65}$ wurde das $\mathrm{mEV}$ erfasst.

Die Hörgerätekontrolle beinhaltete eine Sichtprüfung und die Rückkopplungsprovokation. Außerdem wurde durch Fachpersonal (Hörakustiker) geprüft, ob Hörgeräte- und Versorgungstyp dem Hörverlust angemessen war. Die Überprüfung der Verstärkung erfolgte über In-situ-Messungen. Der Sprachtest mit Hörgerät erfolgte im Freifeld bei $65 \mathrm{~dB}$ SPL seitengetrennt für das linke und rechte Ohr. Die kontralaterale Seite wurde adäquat durch Ohrstöpsel geblockt.

\section{Datenanalyse}

Die audiometrischen Daten der 740 Ohren wurden für alle Patienten seitengetrennt analysiert. Fälle wurden entsprechend der Ausprägung ihres Hörverlusts in die 10 Audiogrammtypen $\mathrm{N}_{1}-\mathrm{N}_{7}$ und $\mathrm{S}_{1}-\mathrm{S}_{3}$ nach Bisgaard [1] mithilfe des minimalen euklidischen Abstands eingeteilt.

Zum Vergleich von $\mathrm{mEV}$ und $\mathrm{EV}_{65}$ (HG) wurden sowohl Differenz

$$
D=m E V-E V_{65}(H G)
$$


als auch der der Quotient

$$
Q=\frac{E V_{65}(H G)}{m E V}
$$

aus beiden Größen berechnet.

Der Quotient Q steht für den Anteil des $\mathrm{mEV}$, der mit dem Hörgerät in Sprachverstehen bei $65 \mathrm{~dB}$ SPL umgesetzt werden kann. Er kann als sprachaudiometrischer Wirkungsgrad einer Hörgeräteversorgung interpretiert werden.

Die logistische Regressionsanalyse des Sprachverstehens, mEV bzw. $\mathrm{EV}_{65}(\mathrm{HG})$, in Abhängigkeit vom Hörverlust, 4FPTA, wurde analog zu Hoppe et al. [7] durchgeführt.

Zur Prüfung der Gruppentrends innerhalb der beiden Audiogrammgrundtypen wurde der nichtparametrische Jonckheere-Terpstra-Test verwendet [4]. Die statistischen Tests wurden mit SPSS V24 (Fa. IBM, Armonk, NY, USA) durchgeführt, die Abbildungen wurden mit Matlab $^{\circledR}$ R2017a (Fa. Mathworks, Natick, MA, USA) erstellt.

\section{Ergebnisse}

\section{Klassifikation der Audiogramme}

Die 10 Standardtypen nach Bisgaard [1] sind in Abb. 1 dargestellt. Die Ergebnisse der hier vorgenommenen Zuordnung sind durch die Fallzahlen $n$ rechts neben den Kurven repräsentiert.

In - Tab. 1 sind die Gruppengrößen und statistische Größen für Alter und 4FPTA zu jedem Audiogrammtyp nach erfolgter Klassifikation der 740 Fälle zusammengefasst. Das mittlere Alter der Einzelgruppen variiert von 50 Jahren bis 68 Jahren.

Die Abb. 2 stellt das monaurale Sprachverstehen der Hörgerätenutzer als Boxplots in Abhängigkeit vom 4FPTA und Audiogrammtyp dar. Im oberen Teil a sind die Ergebnisse der Messung mit Kopfhörern bei 65 dB SPL, im mittleren Teil b die im Freifeld mit Hörgeräten bei $65 \mathrm{~dB}$ SPL gemessenen Werte dargestellt. Der untere Teil c zeigt den Gewinn durch die Hörgeräteversorgung als Differenz der beiden Messungen. Die Daten sind entsprechend den dazugehörigen Audiogrammen mit schwarzen Boxplots

HNO https://doi.org/10.1007/s00106-019-00780-5

(c) Springer Medizin Verlag GmbH, ein Teil von Springer Nature 2019

\author{
C. Dörfler · T. Hocke · A. Hast · U. Hoppe
}

\title{
Sprachverstehen mit Hörgeräten für 10 Standardaudiogramme
}

\section{Zusammenfassung}

Hintergrund. Die Verbesserung des Sprachverstehens in Ruhe ist primäres Ziel einer Hörgeräteversorgung. In der Praxis variiert der Versorgungserfolg sehr stark. Ziel dieser Arbeit war die Untersuchung des Zusammenhangs zwischen Ausmaß und Art des Hörverlusts (Audiogrammtyp), maximalem Einsilberverstehen und Sprachverstehen mit Hörgerät.

Material und Methoden. Es wurden die tonund sprachaudiometrischen Werte von 740 Ohren von 370 Patienten ausgewertet, die sich zwischen 2012 und 2017 in unserem Hörzentrum zur Kontrolle ihrer Hörgeräteversorgung vorgestellt hatten. Das maximale Verstehen im Freiburger Einsilbertest (mEV) und das Einsilberverstehen mit Hörgerät, $\mathrm{EV}_{65}(\mathrm{HG})$, wurden für 10 unterschiedliche Standardaudiogrammtypen analysiert. Ergebnisse. Das $E_{65}(\mathrm{HG})$ für unterschiedliche Hörverlustklassen liegt innerhalb der Fehlergrenzen einer früheren Untersuchung und weist eine Differenz von 10-20 Pro- zentpunkten zum mEV auf. Diese Differenz ist bei flachen und moderat abfallenden Hörverlusten tendenziell größer als bei steil abfallenden Audiogrammen. Der Quotient $\mathrm{EV}_{65}(\mathrm{HG}) / \mathrm{mEV}$ kann als Wirkungsgrad der Hörgeräteversorgung interpretiert werden, da er das tatsächlich erreichte Sprachverstehen mit Hörgerät auf das maximal erreichbare Einsilberverstehen des Schwerhörigen bezieht.

Schlussfolgerung. Die Erwartungshaltung des Schwerhörigen bezüglich einer Hörgeräteversorgung ist unter Berücksichtigung des maximalen Einsilberverstehens und der abgeleiteten Qualitätsmaße auf Ausmaß und Frequenzverlauf des Hörverlusts abzustimmen.

Schlüsselwörter

Maximales Einsilberverstehen · Sprachaudiometrie $\cdot$ Tonaudiometrie $\cdot$ Sprachverständlichkeit · DIN EN 60118-15

\section{Speech recognition with hearing aids for 10 standard audiograms}

\section{Abstract}

Background. Improvement of speech perception in quiet is an important goal of hearing aid provision. In practice, results are highly variable. The aim of this study was to investigate the relationship between type and extent of hearing loss (audiogram type), maximum word recognition score, and aided speech perception.

Materials and methods. Pure tone and speech audiometric data of 740 ears in 370 patients were reviewed. All subjects visited our hearing center for hearing aid evaluation between 2012 and 2017. The maximum word recognition score WRS $_{\max }$ ) and the monosyllabic speech recognition score with hearing aids, WRS $65(\mathrm{HA})$ were analyzed for 10 different standard audiogram types.

Results. The $\mathrm{WRS}_{65}(\mathrm{HA})$ with hearing aids for different degrees of hearing loss is, within error boundaries, comparable to previous investigations and shows a difference of 10-20 percentage points to the $W_{R S}$ max. This difference tends to be larger for flat and moderately sloping audiograms compared to steep-sloping audiograms. The ratio WRS $_{65}(\mathrm{HA}) / \mathrm{WRS}_{\max }$ can be interpreted as an efficiency factor for hearing aid provision, since it relates speech recognition with hearing aids to the maximally achievable information carrying capacity of the hearing impaired.

Conclusion. The expectation regarding hearing aid provision has to be adjusted according to maximum word recognition score, the derived quality measures, degree of hearing loss, and audiogram type.

\section{Keywords}

Maximum word recognition score $\cdot$ Speech audiometry - Tone audiometry . Speech comprehensibility · IEC 60118-15 

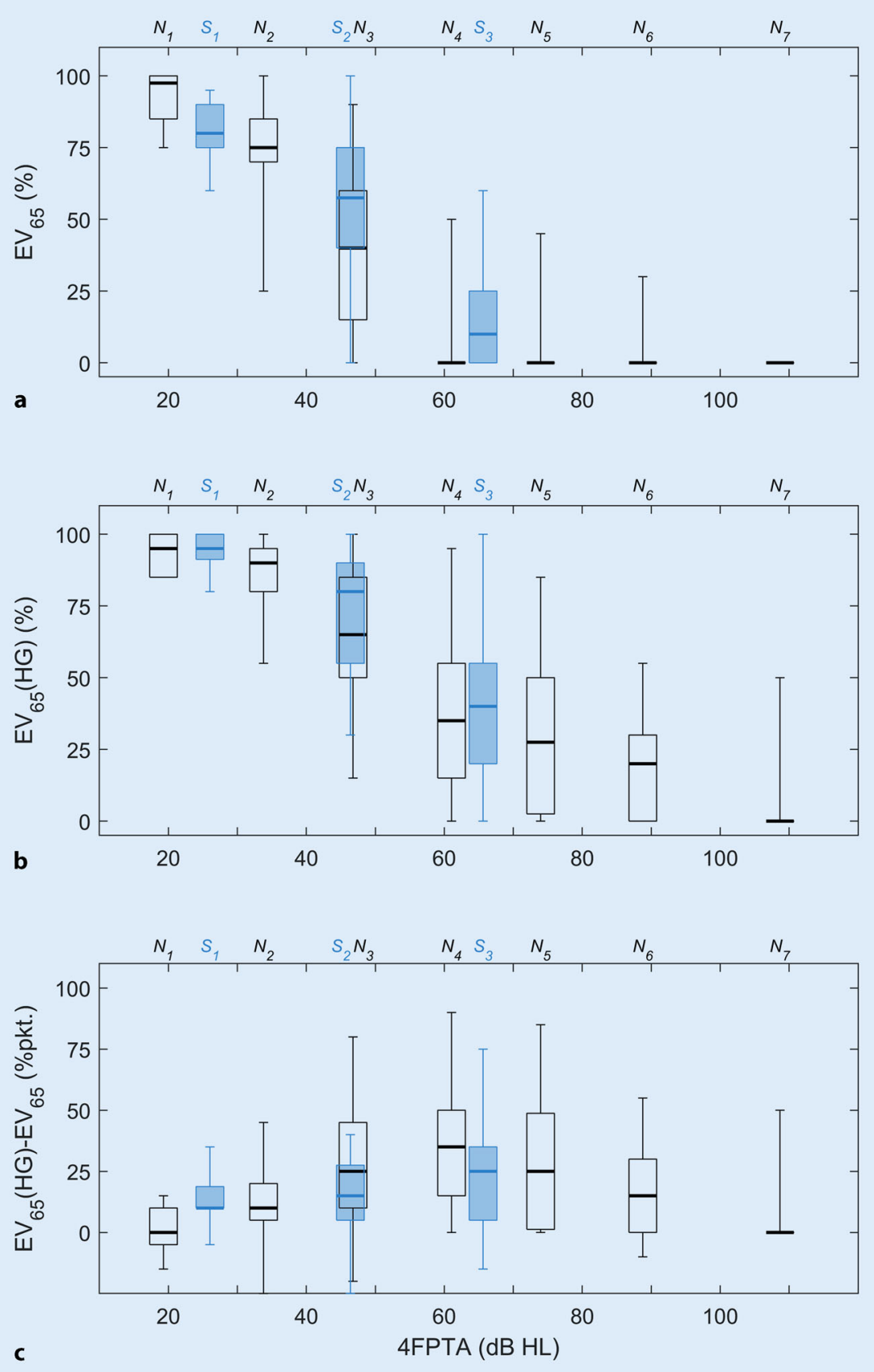

Abb. 2 \ Monaurales Sprachverstehen für die 10 Audiogrammtypen (schwarz N-Typ, blau S-Typ) ohne (a) und mit Hörgerät (b) sowie die zugehörige Differenz (c) in Abhängigkeit vom 4FPTA. Die Messungen ohne Hörgerät erfolgten unter Kopfhörern bei $65 \mathrm{dBSPL}$, die Hörgerätemessungen im Freifeld bei 65 dB SPL. Die Boxplots beinhalten den Median, das erste und dritte Quartil sowie Minimum und Maximum. Diex-Position eines Boxplots entspricht dem mittleren 4FPTA der Fälle, die dem entsprechenden Audiogrammtyp zugeordnet worden sind, s. auch •Tab. 1

für den N-Typ bzw. blauen Boxplots für den S-Typ zusammengefasst.

Für geringgradige Hörverluste $\left(\mathrm{N}_{1}, \mathrm{~N}_{2}\right.$ und $\mathrm{S}_{1}$ ) wird mit Hörgeräten immer ein $\mathrm{EV}_{65}(\mathrm{HG})$ größer als $50 \%$ erreicht. Für die mittelgradigen Hörverluste $\left(\mathrm{N}_{3}\right.$ und $S_{2}$ ) wird dies in 3 Viertel der Fälle erreicht.
Einzelfällen beobachtet, 4 von 71 in der $\mathrm{N}_{6}$-Gruppe und 1 von 80 in der $\mathrm{N}_{7}$-Gruppe. Wie in Abb. $2 c$ dargestellt, finden sich für Hörverluste zwischen 40 und $90 \mathrm{~dB}$ im Mittel die größten Verbesserungen durch die Hörgeräteversorgung. Allerdings zeigt sich in 5,5\% der Fälle ein in der Freifeldmessung mit Hörgerät gegenüber der Kopfhörermessung reduziertes Sprachverstehen. Diese Verschlechterung lag zwischen 5 und 25 Prozentpunkten.

Die - Abb. 3 setzt das gemessene maximale Einsilberverstehen in Beziehung zu dem mit Hörgerät erreichten Einsilberverstehen. Hierfür wurde zunächst das $\mathrm{mEV}$ für die einzelnen Gruppen in - Abb. 3a dargestellt. Die - Abb. 3b, c stellen jeweils die Differenz $D$ und den Quotienten $Q$ in Abhängigkeit vom Audiogrammtyp dar. Bei Werten für $D$ nahe 0 und $Q$ nahe 1 wird das $m E V$ durch das $\mathrm{EV}_{65}(\mathrm{HG})$ angenähert. Die $\mathbf{A b b}$. 3a zeigt die mit zunehmenden Hörverlusten einhergehende Verringerung des maximalen Einsilberverstehens. Die in - Abb. 3b dargestellten Differenzen ergeben ein ähnliches, durch Sättigungseffekte des Tests geprägtes Bild wie in - Abb. 2c. Die Berechnung der Quotienten $Q$ ist nur für die Fälle mit einem mEV größer 0 möglich. So konnte beispielsweise für die Gruppe des $\mathrm{N}_{6}$-Typs in 60 von 71 Fällen ein $\mathrm{mEV}$ größer 0 bestimmt werden. Die korrespondierenden Angaben für die anderen Gruppen finden sich an der oberen $\mathrm{x}$-Achse von - Abb. 3c. Für die Analyse des Einflusses des Audiogrammtyps auf $Q$ wurden die beiden Grundtypen (N-Typ und S-Typ) getrennt betrachtet. Die Trendanalyse für die Mediane wurde mit dem Jonckheere-Terpstra-Test durchgeführt. Dieser ergibt für beide Grundtypen eine signifikante Abhängigkeit des Wirkungsgrads $Q$ vom Ausmaß des Hörverlusts. Die Teststatistik J, die standardisierte Teststatistik $\mathrm{z}$ und der entsprechende $p$-Wert liegen bei $\mathrm{J}=36.468 ; \mathrm{z}=-11,7$; $p<0,001$ für N-Typ Audiogramme bzw. $\mathrm{J}=1020 ; \mathrm{z}=-5,8 ; p<0,001$ für $\mathrm{S}$-Typ Audiogramme.

Die Abb. 4 setzt die aktuellen Ergebnisse in Relation zu den 2011 und 2012 erhobenen Daten [7]. Die grauen Bereiche in der Abbildung repräsentieren das 

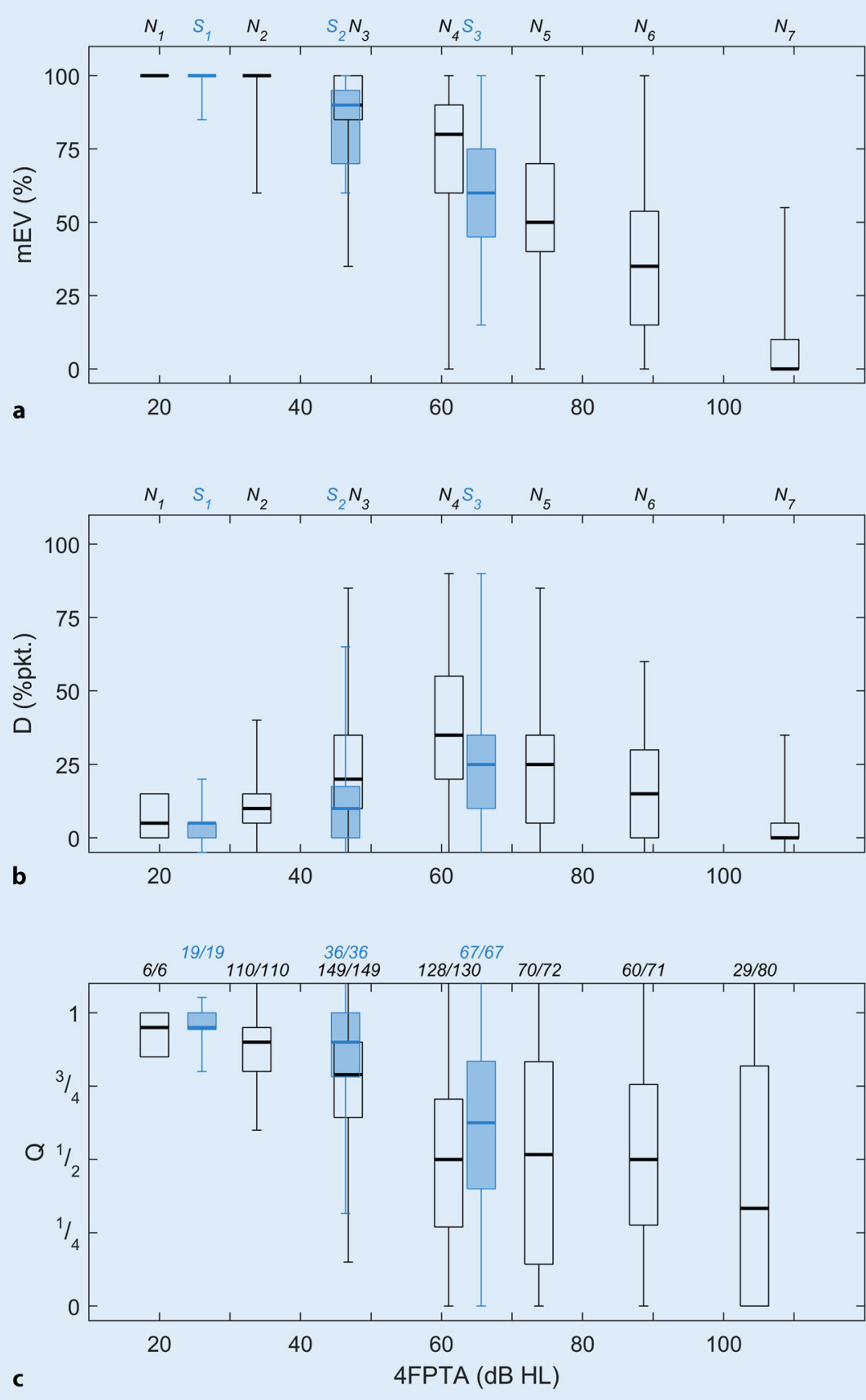

Abb. 3 ॥ Maximales Einsilberverstehen (a) und die Relation zum Sprachverstehen mit Hörgerät für die 10 Audiogrammtypen (schwarz N-Typ, blau S-Typ) in Abhängigkeit vom 4FPTA. Dies ist sowohl als Differenz (b) als auch als Verhältnis (c) beider Messungen dargestellt. Die Messung des mEV erfolgte unter Kopfhörern, die Hörgerätemessung im Freifeld bei $65 \mathrm{dBSPL}$. Die Zuordnung der Boxplots zu den Audiogrammtypen nach - Abb. 1 ist durch die obere Beschriftung gegeben. Die Boxplots beinhalten den Median, das erste und dritte Quartil sowie Minimum und Maximum. Diex-Position eines Boxplots entspricht dem mittleren 4FPTA der Fälle, die dem entsprechenden Audiogrammtyp zugeordnet worden sind. Im unteren Teil (c) können nur die Fälle mit einem $m E V>0 \%$ dargestellt werden. Die Anteile dieser Fälle in den Gruppen $\mathrm{N}_{1-7}$ und $\mathrm{S}_{1-3}$ sind jeweils oberhalb des Boxplots (c) angegeben
95\%-Konfidenzintervall der logistischen Regression der Referenzdaten [7], dunkelgrau für das mEV und hellgrau für das $\mathrm{EV}_{65}(\mathrm{HG})$. Dargestellt sind die Ergebnisse der logistischen Regression des $\mathrm{mEV} b z w . \mathrm{EV}_{65}(\mathrm{HG})$, in Abhängigkeit vom Hörverlust für die Audiogramme des N-Typs und des S-Typs. In die Regression für den N-Typ gingen 618 Fälle (Ohren) ein, für den S-Typ 122 Fälle. Während die Anpassfunktion für das mEV der Gruppen des N-Typs jeweils im mittleren Bereich des 95\%-Konfidenzintervalls liegt, findet sich bei den Gruppen des S-Typs für das mEV ein Fit unterhalb des 95\%-Konfidenzintervalls der logistischen Regression. Für das Sprachverstehen mit Hörgeräten zeigt sich ein anderes Bild: Die beiden Regressionsfunktionen für das $\mathrm{EV}_{65}(\mathrm{HG})$ liegen weitgehend innerhalb des 95\%-Konfidenzintervalls mit einer Tendenz hin zu besseren Werten für die Gruppen des S-Typs gegenüber Gruppen des N-Typs für gering- und mittelgradige Hörverluste.

\section{Diskussion}

Ziel der Arbeit war es, die im Rahmen der Hörgeräteüberprüfung erhobenen tonund sprachaudiometrischen Größen zueinander in Beziehung zu setzen. Im Mittelpunkt stand dabei das Sprachverstehen in Ruhe, die Verbesserung durch ein Hörgerät als der „Hauptwirkung einer Hörgeräteversorgung“ [24] sowie die Beziehung zum maximalen Einsilberverstehen. In Ergänzung zu früheren Arbeiten [7, 8] wurden die Fälle entsprechend dem Audiogrammverlauf jeweils den verschiedenen Audiogrammtypen nach Bisgaard [1] zugeordnet. Bei gleichem 4FPTA ist das Einsilberverstehen mit Hörgerät bei stark frequenzabhängigen Hörverlusten höher als bei moderat abfallenden Audiogrammen.

\section{Spezifische Audiogrammverläufe und Sprachverstehen}

Das Einsilberverstehen bei $65 \mathrm{~dB}$ mit und ohne Hörgerät ist primär durch den 4FPTA bestimmt. Für das $\mathrm{EV}_{65}(\mathrm{HG})$ liefert die Berücksichtigung des Audiogrammtyps zusätzliche Informationen (• Abb. 2b). Das $\mathrm{EV}_{65}(\mathrm{HG})$ fällt sowohl 


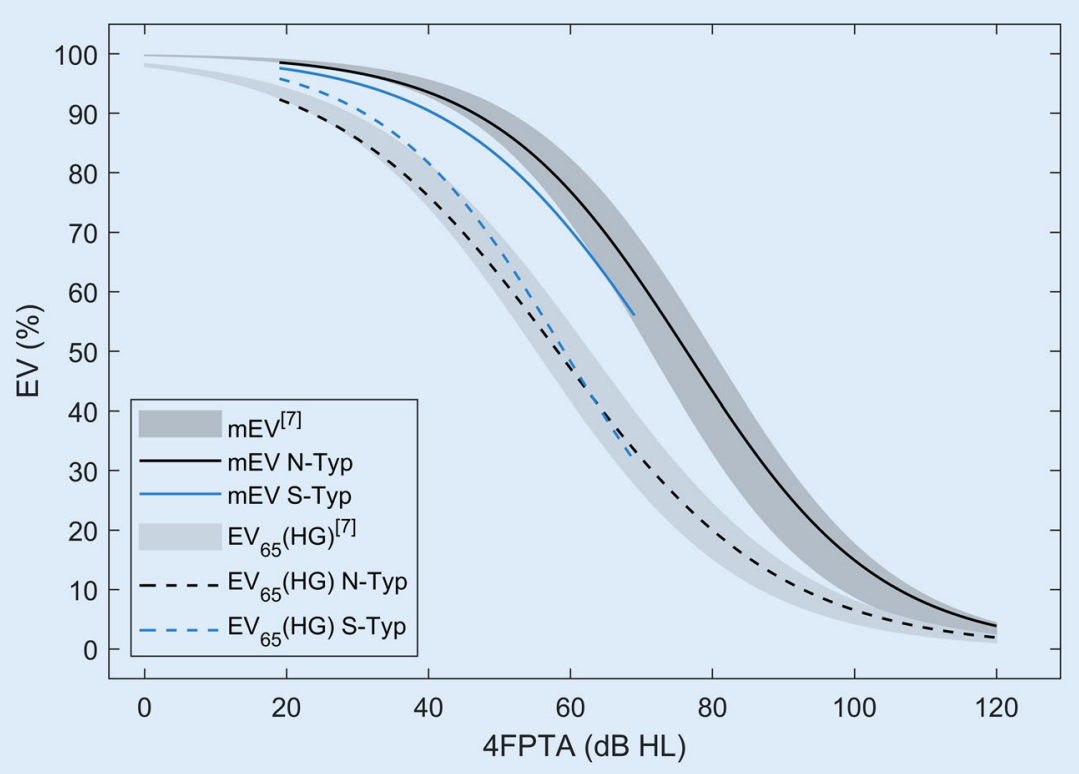

Abb. $4 \Delta$ Logistische Regression des Einsilberverstehens (EV), mEV bzw. $\mathrm{EV}_{65}(\mathrm{HG})$, in Abhängigkeit vom Hörverlust, 4FPTA für N- und S-Typ-Audiogramme nach Bisgaard et al. [1]. Die grauen Bereiche stellen das 95\%-Konfidenzintervall der logistischen Regression nach Hoppe et al. [7] dar. Die schwarzen Linien zeigen die Ergebnisse der Regression für die aktuellen Daten der N-Typ-Audiogramme, die blauen Linien stehen für die S-Typ-Audiogramme

in den $\mathrm{N}$ - und S-Typen mit wachsendem 4FPTA monoton ab. Jedoch liegt die Regressionsfunktion für die Versorgungen von Patienten mit S-Typ-Audiogrammen oberhalb der Regressionsfunktion für die mit N-Typ-Audiogrammen. Insbesondere zeigt der Vergleich der $\mathrm{N}_{3}$ - und $\mathrm{S}_{2}$-Gruppe mit nahezu gleichem 4FPTA und annähernd gleicher Altersverteilung, dass das $\mathrm{EV}_{65}(\mathrm{HG})$ für die S-TypAudiogramme um 15 Prozentpunkte oberhalb des $\mathrm{EV}_{65}(\mathrm{HG})$ für die N-TypAudiogramme liegt. Die Ergebnisse der logistischen Regression unterstützen diesen Befund: Bei gering- bis mittelgradigen Schwerhörigkeiten lässt sich insbesondere bei steil abfallenden Audiogrammen mit Hörgeräten ein gutes Sprachverstehen erzielen. Während in den S-Gruppen bei der Messung des $\mathrm{mEV}$ über Kopfhörer der steile Abfall im Audiogramm augenscheinlich $\mathrm{zu}$ schlechteren Ergebnissen führt, gelingt es bei der Hörgeräteversorgung besser, die frequenzspezifische Abschwächungskomponente des Hörverlusts auszugleichen. Für die N-Gruppen entspricht die frequenzunabhängige Verstärkung bei der mEV-Bestimmung also eher der Hörgeräteanpassung als für die S-Grup- pen, wo tiefe Frequenzen nicht oder nur verstärkt werden müssen. Dies erklärt auch z. T. die in $\bullet$ Abb. 3 c dargestellten Unterschiede in der Annäherung des $\mathrm{mEV}$ durch das $\mathrm{EV}_{65}(\mathrm{HG})$. Eine weitere Erklärung könnte die gerade in den letzten Jahren zunehmend mögliche offene Versorgung sein, für die gerade bei den S-Typ-Audiogrammen gute tonaudiometrische Voraussetzungen vorliegen [11]. Ein zunächst niedrigeres $\mathrm{mEV}$ der S-Gruppe kann durch die mit einer offenen Versorgung einhergehenden Vorteile $[3,20]$ kompensiert werden.

\section{Vergleich mit früheren Untersuchungen}

In $\bullet$ Abb. 4 sind die Ergebnisse der logistischen Regression für die N- und S-Typen dargestellt. Für eng umschriebene 4FPTA-Bereiche lassen sich Unterschiede für die N- und S-Typ-Audiogramme nachweisen. Jedoch lassen die in der Regression gefundenen Unterschiede vor dem Hintergrund der ungleich höheren individuellen Variabilität keine größeren klinischen Konsequenzen erwarten. Demzufolge erscheint die Reduktion des tonaudiometrischen Hörverlusts auf den 4FPTA zur Beschreibung der Zusammenhänge mit den Messungen des Sprachverstehens zumindest für große Patientengruppen und den daraus abgeleiteten Aussagen angebracht $[7,8,12$, $15,21]$.

Die in Abb. 4 vorgenommene Gegenüberstellung des mit Hörgerät erzielten Sprachverstehens mit einer in vergleichbarer Population durchgeführten früheren Analyse für den Zeitraum von Januar 2011 bis Juli 2012 [7] ergibt Unterschiede im Bereich weniger Prozentpunkte. Demnach werden für das Einsilberverstehen keine Unterschiede zu den heutigen Ergebnissen gefunden. Dieser Befund steht in Übereinstimmung mit einer aktuellen Studie [2] zur subjektiven Hörqualität.

\section{Praktische Aspekte der Hörgeräteevaluierung mittels Sprachaudiometrie}

Ein weiterer Aspekt der vorliegenden Arbeit war die weitergehende Beschreibung des Zusammenhangs zwischen $\mathrm{mEV}$ und $\mathrm{EV}_{65}(\mathrm{HG})$ vor dem Hintergrund der sprachaudiometrischen Evaluierung einer Hörgeräteversorgung. In der Hilfsmittelrichtlinie des Gemeinsamen Bundesausschusses [5] wird ein Gewinn des $\mathrm{EV}_{65}(\mathrm{HG})$ gegenüber dem $\mathrm{EV}_{65}$ von mindestens 20 Prozentpunkten gefordert. Weiterhin wird dort die möglichst große Annäherung des $\mathrm{EV}_{65}(\mathrm{HG})$ an das $\mathrm{mEV}$ gefordert, auch wenn dies häufig nicht erreicht wird [7, 8, 12, 15, 21]. Die Hauptgründe hierfür können mangelnde Akzeptanz der erforderlichen akustischen Verstärkung, zu geringe Restdynamik, eine im Alter reduzierte Anpassung an die apparative Versorgung oder technische Ursachen sein $[3,7,21$, 26].

Lediglich für Hörverluste unterhalb $40 \mathrm{~dB}$ und oberhalb $100 \mathrm{~dB}$ liegen die Differenzen zwischen $\mathrm{mEV}$ und $\mathrm{EV}_{65}(\mathrm{HG})$ für die Mehrheit der Versorgungen unterhalb 20 Prozentpunkten. Für diese Bereiche limitieren die bekannten Floor- und Ceilingeffekte des verwendeten Sprachtests die Differenz bzw. ihren diagnostischen Wert.

Bei gering- bis mittelgradigen Hörverlusten kann für die überwiegende 
Tab. 2 Sprachaudiometrische Kennwerte für 4 Fälle, bei denen das Einsilberverstehen mit Hörgerät 10 Prozentpunkte unterhalb des $m E V$ liegt. Der sprachaudiometrische Wirkungsgrad Q variiert in diesen Fällen zwischen 0 und 0,9

\begin{tabular}{l|l|l|l|l}
\hline Beispiel & mEV & EV $_{\mathbf{6 5}}(\mathbf{H G})$ & D & $\mathbf{Q}$ \\
\hline$\# 1$ & 100 & 90 & 10 & 0,9 \\
$\# 2$ & 50 & 40 & 10 & 0,8 \\
$\# 3$ & 25 & 15 & 10 & 0,6 \\
\hline 4 & 10 & 0 & 10 & 0
\end{tabular}

Mehrzahl der Versorgungen ein Wirkungsgrad von mehr als $3 / 4$ erzielt werden. $\mathrm{Ab}$ einer hochgradigen Schwerhörigkeit (4FPTA $>60 \mathrm{~dB}$ ) fällt dieser auf $1 / 2 \mathrm{ab}$. Dass der Wirkungsgrad für den Fall von $\mathrm{mEV}=0 \%$ nicht definiert ist, ist hierbei von geringer praktischer Relevanz. In diesen Fällen sollten alternative Sprachtests in Erwägung gezogen werden. Ist dies nicht möglich, so ist dann die Sprachaudiometrie ohnehin keine angezeigte Methode zur Evaluierung der Hörgeräteversorgung.

Die - Tab. 2 verdeutlicht anhand von 4 Beispielen mit identischer Differenz die Bedeutung des Wirkungsgrads Q: Wird mit dem Hörgerät das maximale Einsilberverstehen erreicht, so sind $\mathrm{mEV}$ und $\mathrm{EV}_{65}(\mathrm{HG})$ identisch. Die Differenz ist 0 und der Wirkungsgrad 1. Relevante Unterschiede ergeben sich erst, wenn das mEV nicht erreicht wird. Der Wirkungsgrad $Q$ quantifiziert den Anteil des erreichten vom maximal möglichen Sprachverstehens (informationstragende Kapazität $[6,9]$ ) und kann in Ergänzung zur Differenz für die Charakterisierung einer Hörgeräteversorgung herangezogen werden.

Inwieweit diese an einer HNO-Klinik mit angeschlossenem Hör- und CIZentrum gefundenen Ergebnisse repräsentativ für die Hörgeräteversorgung in der Fläche sind, kann auch über eine Bestätigung durch andere Arbeiten [12, $15,18,19,21]$ nicht gesagt werden, da diese in einem vergleichbaren Umfeld entstanden sind. Daher wären versorgungsnahe Studien zum aktuellen Stand der Hörgeräteversorgungen auch außerhalb großer Hörzentren wünschenswert. Zur Vergleichbarkeit der Ergebnisse sollten diese Untersuchungen sprachaudiometrischen Standards entsprechen, wie sie für klinische Studien zur Hörverbesserung [22], z. B. mit aktiven Mittel- ohrimplantaten [16], vorgeschlagen wurden. Diese sollten möglichst auch Sprachverständlichkeitsmessungen im Störgeräusch einschließen.

\section{Fazit für die Praxis}

- Der sprachaudiometrische Wirkungsgrad einer Hörgeräteversorgung, berechnet als Quotient aus Sprachverstehen mit Hörgerät, $\mathrm{EV}_{65}(\mathrm{HG})$, und maximalem Einsilberverstehen, $\mathrm{mEV}$, liefert zusätzliche Informationen.

- Der Wirkungsgrad einer Hörgeräteversorgung liegt im Mittel bei stark frequenzabhängigen Audiogrammen vom S-Typ höher als bei den flacher verlaufenden Audiogrammen vom N-Typ.

- Für Hörverluste unterhalb $60 \mathrm{~dB}$ HL liegt der Wirkungsgrad bei nahe 1 . Oberhalb $60 \mathrm{~dB}$ werden im Mittel Werte von nur $1 / 2$ erreicht.

\section{Korrespondenzadresse}

\section{Prof. Dr. Dr. U. Hoppe}

Audiologische Abteilung und $\mathrm{Cl}$ Centrum CICERO Hals-Nasen-Ohrenklinik, Universitätsklinikum Erlangen Waldstraße 1, 91054 Erlangen, Deutschland Ulrich.Hoppe@uk-erlangen.de

\section{Einhaltung ethischer Richtlinien}

Interessenkonflikt. C. Dörfler, T. Hocke, A. Hast und $U$. Hoppe geben an, dass kein Interessenkonflikt besteht.

Alle beschriebenen Untersuchungen am Menschen wurden mit Zustimmung der zuständigen Ethik-Kommission, im Einklang mit nationalem Recht sowie gemäß der Deklaration von Helsinki von 1975 (in der aktuellen, überarbeiteten Fassung) durchgeführt. Von allen beteiligten Patienten liegt eine Einverständniserklärung vor. Die Studie wurde von der Ethik-Kommission der Friedrich-Alexander-Universität Erlangen positiv beschieden (Nr. 162 17 BC).

\section{Literatur}

1. Bisgaard N, Vlaming MS, Dahlquist M (2010) Standard audiograms for the IEC 60118-15 measurement procedure. Trends Amplif 14:113-120

2. Braun B, Dietrich A, Akcicek B et al (2015) Hörgeräteversorgung. Folgen der Festbetragsverdoppelung auf Compliance, Ergebnisqualität und Zuzahlungen. HNO 63:850-856

3. Dillon H (2012) Hearing aids, 2. Aufl. Thieme, New York, Stuttgart

4. Field A (2013) Discovering statistics using IBM SPSS statistics, 4. Aufl. SAGE, Los Angeles

5. Gemeinsamer Bundesausschuss (2018) Hilfsmittel-Richtlinie über die Verordnung von Hilfsmitteln in der vertragsärztlichen Versorgung (HilfsmittelRichtlinie/HilfsM-RL) in der Neufassung vom 19. Juli 2018. BAnz AT 02.10.2018 B2. Gemeinsamer Bundesausschuss, Berlin

6. Halpin C, Rauch SD (2008) Clinical implications of a damaged cochlea: puretone thresholds vs information carrying capacity. Otolaryngol Head Neck Surg 140:473-476

7. Hoppe U, Hast A, Hocke T (2014) Sprachverstehen mit Hörgeräten in Abhängigkeit vom Tongehör. HNO 62:443-448

8. Hoppe U, Hast A, Hocke T (2015) Audiometrybased screening procedure for cochlear implant candidacy. Otol Neurotol 36(6):1001-1005

9. Hoppe U, Hocke T, Müller A, Hast A (2016) Speech perception and information-carrying capacity for hearing aid users of different ages. Audiol Neurotol 21(suppl 1):16-20

10. Hoppe U, Hesse G (2017) Hörgeräte: Indikationen, Technologie, Anpassung und Qualitätskontrolle. Laryngorhinootologie 96(S1):43-65

11. Kießling J (2018) Konventionelle Hörsysteme: Hörgeräte.In:Kießling J, Kollmeier B, Baumann U(Hrsg) Versorgung mit Hörgeräten und Hörimplantaten, 3. Aufl. Thieme, Stuttgart

12. Kronlachner M, Baumann U, Stöver T, Weißgerber T (2018) Untersuchung der Qualität der Hörgeräteversorgung bei Senioren unter Berücksichtigung kognitiver Einflussfaktoren. Laryngorhinootologie 97:852-859

13. Lesica NA (2018) Why do hearing aids fail to restore normal auditory perception. Trends Neurosci 41:174-185

14. Löhler J, Akcicek B, Wienke A, Hoppe U (2014) Komplikationen bei der Hörgeräteversorgung ohneHNO-Arzt.HNO 62:360-366

15. Maier H, Lenarz T, Dollezal LV, Busch S (2018) Direct acoustic cochlear implants lead to an improved speech perception gap compared to conventional hearing aid. Otol Neurotol 39:1147-1152

16. Maier H, Baumann U, Baumgartner WD et al (2018) Minimal reporting standards for active middle ear hearing implants. Audiol Neurootol 23:105-115

17. Mathers C, Smith A, Concha M (2000) Global burden of hearing loss in the year 2000. Global burden of disease. World Health Organization, Geneva, S130

18. McRackan TR, Ahlstrom JB, Clinkscales WB et a (2016) Clinical implications of word recognition differences in earphone and aided conditions. Otol Neurotol 37:1475-1481

19. McRackan TR, Fabie JE, Burton JA et al (2018) Earphone and aided word recognition differences in cochlear implant candidates. Otol Neurotol 39:e543-e549

20. Mueller HG, Ricketts TA (2006) Open-canal fittings: ten take-home tips. Hear J59:24-39

21. Müller A, Hocke T, Hoppe U, Mir-Salim P (2016) Der Einfluss des Alters bei der Evaluierung des 


\section{Originalien}

funktionellen Hörgerätenutzensmittels Sprachaudiometrie. HNO 64:143-148

22. Müller J, Plontke SK, Rahne T (2017) Sprachaudiometrische Zielparameter in klinischen Studien zur Hörverbesserung. HNO 65:211-218

23. Schuchardt W, Löhler J (2012) Hörgeräteversorgung ohneHNO-Arzt? HNO 60:823-826

24. Steffens T, Marcrum SC (2018) Fachärztliches Basiswissen zur Wirksamkeit von Hörgeräten in Abhängigkeit der Art und Pathophysiologie einer Hörstörung. HNO 66:122-127

25. von Gablenz P, Holube I (2015) Prävalenz von Schwerhörigkeit im Nordwesten Deutschlands: Ergebnisse einer epidemiologischen Untersuchung zum Hörstatus (HÖRSTAT). HNO 63:195-214

26. ZwartenkotJW, SnikAFM, Mylanus EAM, Mulder JJS (2014) Amplification options for patients with mixed hearing loss. Otol Neurotol 35:221-226 
Ich bedanke mich bei denjenigen Personen, die mich bei der Anfertigung meiner Doktorarbeit, sowie während meines Studiums unterstützt haben.

Besonderen Dank widme ich meinem Doktorvater Herrn Prof. Dr. - Ing. Dr. rer. med. Ulrich Hoppe für die bereichernde und fachkompetente Zusammenarbeit, die Unterstützung, sowie die stets konstruktive Rückmeldung bezüglich Arbeitsinhalten. Ich hätte mir keine bessere Betreuung beim Erstellen meiner Doktorarbeit vorstellen können.

Auch bedanke ich mich bei den Mitarbeitern des Erlanger Cochlear-Implant-Centrums, ohne die das Auswerten der Daten in dieser Form nicht möglich gewesen wäre.

Meiner Familie danke ich von Herzen für deren uneingeschränkte Unterstützung und deren fachliche Ratschläge während meiner gesamten Studienzeit. Danke Mira für deinen Rückhalt und deine Motivation. Ebenso danke ich Marius, ohne den die Studienzeit in Erlangen nicht ansatzweise so amüsant gewesen wäre. 